\title{
DAS GRUNDSATZPROGRAMM DER AFD UND SEINE HISTORISCHEN PARALLELEN EINE PERSPEKTIVE DER POLITOLINGUISTIK
}

\section{Einleitung: Sprache und Politik}

Das alles führt zu ungerechten Pauschalverdächtigungen gegenüber der Mehrzahl der rechtstreuen, integrierten ausländischen Mitbürger als auch der rechtstreuen Asylbewerber. (AfD 2016, S. 42)

Der gesetzliche Mindestlohn [...] korrigiert im Bereich der Entlohnung die Position der Niedriglohnempfänger als schwache Marktteilnehmer gegenüber den Interessen der Arbeitgeber als vergleichsweise starke Marktteilnehmer. (AfD 2016, S. 24f.)

Weder familienbedingte Erwerbspausen noch Teilzeitarbeit sollten sich negativ auf den beruflichen Status und die weitere berufliche Entwicklung auswirken. (AfD 2016, S. 34)

Das sind Sätze aus dem Grundsatzprogramm der AfD (Vorlage zum Bundesparteitag am 30.04.2016/01.05. 2016). Sie drücken eine Einstellung und Haltung aus, die jeden Freund und jede Freundin von Multikulturalität, Familienfreundlichkeit und Sozialstaat in höchstem Maß erfreuen.

Die ungeregelte Asylzuwanderung, die dem Wirtschaftsstandort Deutschland nicht nutzt und der Gesellschaft schadet. (AfD 2016, S. 46)

Niedrigqualifizierte wandern überwiegend über missbräuchliche Asylanträge zu [...]. (AfD 2016, S. 51)

Jeder Einwanderer hat eine unabdingbare Bringschuld, sich zu integrieren; (AfD 2016, S. 47)

Mehrzahl der Täter im Bereich der organisierten Kriminalität sind Ausländer. (AfD 2016, S. 19)

Das sind Sätze aus dem Grundsatzprogramm der AfD. Sie drücken eine Einstellung und Haltung aus, die jeden Freund und jede Freundin von nationalstaatlichkonservativen und exkludierenden Konzepten in höchstem Maß erfreuen.

\section{WIDERSPRÜCHLICH ERSCHEINENDE}

\section{AUSSAGEN ALS SPRACHLICH- KOMMUNIKATIVE STRATEGIE DER AFD}

Auf das Ganze des Textes gesehen ist damit eine sprachliche Strategie der Vertreterinnen und Vertreter der AfD demonstriert, die sie zu ihrem sprachlich- kommunikativen Prinzip gemacht haben: mit widersprüchlich erscheinenden Aussagen den Eindruck von Differenziertheit, gerechtem Urteil und sorgfältiger Abwägung erzeugen und damit diskutabel sein für Adressaten unterschiedlicher Provenienz. ${ }^{1}$ So beschreibt das AfD-Programm einerseits Positionen, die durchaus mit denen z. B. der Grünen oder der SPD vereinbar sind. ${ }^{2}$ Andererseits können die Aussagen z. T. nicht anders denn als nationalistisch und rassistisch bezeichnet werden. Dieses Phänomen ruft die Linguistik auf, das Programm der AfD mit ihren Mitteln, den Mitteln der ,Sprache und Politik'-Forschung, zu analysieren und $\mathrm{zu}$ beschreiben.

\section{POLITIK FUNKTIONIERT DURCH UND MITTELS SPRACHE}

Die Erkenntnis, dass Politik durch Sprache funktioniert, hat seit 1945, mit der Totalitarismuserfahrung 1933 ff. im Rücken, eine linguistische Perspektive entwickelt, die als ,Sprache und Politik'-Forschung seit den 1960er Jahren eine etablierte Teildisziplin der Sprachwissenschaft darstellt. Von den durch Victor Klemperers „LTI" und Sternbergers / Storz / Süskinds „Wörterbuch des Unmenschen“ ausgehenden nichtprofessionellen Anfängen hat sich die Disziplin von der rein wortbezogenen Perspektive der Frühphase über Text- und Diskursanalysen bis hin zur Internetkommunikation sowohl methodisch als auch theoretisch entwickelt. Die seit Anfang der 1960er Jahre zunehmende Professionalisierung des Teilfachs spiegelt nicht nur die Perspektivenvielfalt ihrer Zugriffe, sondern auch die inhaltliche Komplexität und Vielfalt ihrer Gegenstände: NS-Sprache, Deutsch Ost - Deutsch West, Sprache der 68er, Nachrüstungsdebatte, die politische Wende von 1983 und von 1989 / 90, parlamentarische Kommunikation, semantische Kämpfe, Medien, Migration, Atomkraft, Wahlkampf, Wahlplakate, Geschichte politischer Sprache sind nur einige wenige der Gegenstände, die das Forschungsfeld markieren.

Ausgehend von der sprachtheoretischen Voraussetzung, dass Sprache ein „Mittel der Verhaltenssteuerung und der sozialen Integration" (Dieckmann 1975, S. 27) sowie der Kontrolle ist, bestehen die
Die Autorin ist wissenschaftliche Mitarbeiterin am Institut für Deutsche Sprache in Mannheim. 


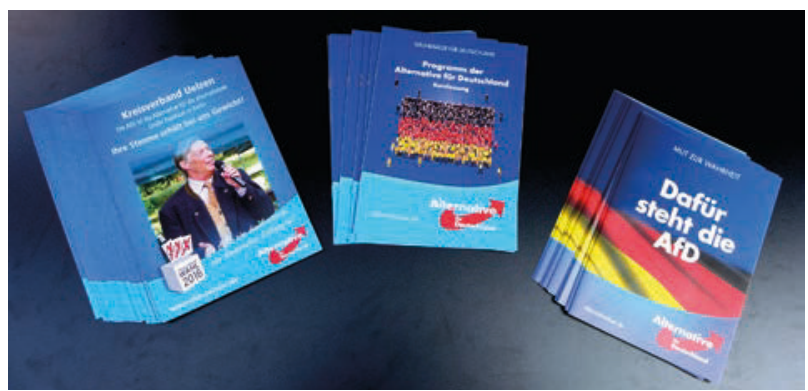

„Dafür steht die AfD“ (Grundsatzprogramm) und weitere Flyer, August (2016)

Ziele von sprachlich Handelnden mit politischer Absicht [...] darin, den Adressaten der Äußerung(en) bestimmte Auffassungen nahezubringen, sie zu bestimmten Überzeugungen zu bringen, bestimmte Einstellungen zu induzieren etc., die sich auf politisch relevante Sachverhalte beziehen. (Liedtke 1996, S. 6)

Aufgabe der nachfolgenden kritischen linguistischen Bewertung des Grundsatzprogramms der AfD ist es, festzustellen, ob bzw. inwiefern es von Geisteshaltungen und Weltsichten bestimmt ist, die dem Regelsystem der im Grundgesetz kodifizierten freiheitlich-demokratischen Gesellschaft widersprechen und den Konsens der Nachkriegsgesellschaft, der seit 1945 also mit den Erfahrungen des Zivilisationsbruchs im Rücken - gilt und der sich in dem einfachen Satz „Die Würde des Menschen ist unantastbar" verdichtet, aufkündigt oder nicht.

\section{IN DEM SATZ „DIE WÜRDE DES MENSCHEN IST UNANTASTBAR“ VERDICHTEN SICH DIE FREIHEITLICH-DEMOKRATISCHEN GRUNDWERTE}

Das Grundsatzprogramm als politische Textsorte Programme politischer Parteien sind Gegenstand der Politolinguistik, seit es diese gibt. Als Textsorte sind Parteiprogramme gekennzeichnet zum einen von ihrer Funktion der Deklaration und Explikation politischer Ziele sowie des Bekenntnisses von Weltsicht, zum andern von ihrem Adressatenbezug, denn sie sind - identitätsstiftend - nach innen auf die Anhänger der Partei (Klein 2008, S. 736) sowie - appellierend - nach außen an die politisch interessierte Gesellschaft gerichtet. ${ }^{3}$

\section{PARTEIPROGRAMME HABEN EINE IDENTITÄTSSTIFTENDE UND APPELLIERENDE FUNKTION}

In den vergangenen Jahren ist, mit der Veränderung des Parteienspektrums, die Auseinandersetzung mit Parteiprogrammen wieder wichtiger geworden. Die Existenz und (durch die Wähler herbeigeführte) Betei- ligung der Piraten-Partei, der Linkspartei und der AfD (seit einem Jahr mit der Abspaltung ALFA) haben das Vierparteiensystem in Frage gestellt. Ein damit einhergehender Zwang zur Profilierung und Abgrenzung kommt insbesondere in den jeweiligen parteiprogrammatischen Schriften zum Ausdruck. Insofern ist insbesondere die Politolinguistik hier herausgefordert, entsprechendes Wissen aus dem Bereich ,Sprache und Politik' zu generieren. Denn: Es wird angesichts der zunehmenden Besetzung politischer Ränder zunehmend wichtiger, die sprachliche Repräsentation politischer Profile zu erkennen, die sprachliche Vergegenwärtigung politischer Strategien zu wissen, zu historischen politischen sprachlichen Vorbildern Bezüge herstellen zu können, Leitwörter und ihre Bedeutungsstruktur zu kennen. Insbesondere diese sind in politischen Programmen insofern von Bedeutung, als die Texte wohl reflektiert sind, Bekenntnischarakter haben und von einer mehr oder weniger großen Parteiöffentlichkeit approbiert sind. Damit sind Parteiprogramme Texte mit einem hohen, der Parteinorm entsprechenden Verbindlichkeitsstatus, der es erlaubt, den Befunden der sprachlichen Analyse eines Parteiprogramms einen hohen Gültigkeitsgrad zuzusprechen.

\section{AUFGABE DER POLITOLINGUISTIK: UNTERSUCHUNG DER SPRACHLICHEN REPRÄSENTATION POLITISCHER}

\section{AUSSAGEN}

Die folgende linguistische Darstellung des Parteiprogramms der AfD stammt aus dem Juni 2016 und ist insofern mitten in der allgemeinen Diskussion dieses Programms situiert. Als es zum Parteitag Ende April / Anfang Mai 2016 nach einer längeren Ankündigungsphase vorgelegt und von der Parteiversammlung mit großer Zustimmung verabschiedet wurde, war die Aufmerksamkeit der Öffentlichkeit groß - und diese hält an.

Die nachfolgende linguistische Beschreibung ist eine politolinguistische Auseinandersetzung mit dem Programm einer Partei, die das nachkriegsdeutsche Partei- 
engefüge verändert hat, die - aufgrund ihrer Positionen, die z. T. als konservativ-national(istisch), z. T. als fremdenfeindlich, z.T. als rassistisch, z.T. als verfassungswidrig bewertet werden - in hohem Maß umstritten ist. Dieses Szenario fordert die Politolinguistik geradezu heraus, mit ihrem Instrumentarium zur Klärung beizutragen.

Insofern politische Positionen entscheidend von dem Selbstbild der diese Positionen vertretenden politischen Instanz geprägt sind, wird zunächst nach Aspekten der Selbstinszenierung der AfD gefragt. Im Anschluss werden Beispiele für ideologische Polysemie Aufschluss darüber geben, in welchen Hinsichten die Autorinnen und Autoren des Programms Leitwörter des gegenwärtigen politischen Diskurses aufnehmen und umdeuten.

Im Sinn einer kulturlinguistischen, d.h. interpretativen, Ableitung von Geisteshaltungen - diese sind im Wesentlichen Gegenstand der öffentlichen Kritik wird anschließend aus spezifischen Formen von Sprachgebrauch im Programm der AfD das Menschenbild dieser Partei herausgearbeitet. Es wird damit vorausgesetzt, dass es sprachlich gefasste Menschenbilder sind, in denen sich politische Orientierungen ausdrücken. Eine solche linguistische Aufbereitung stellt auf empirisch fundierte Weise die Grundlage der hier nicht zu leistenden politischen Beurteilung bereit.

Die Frage nach dem Menschenbild der AfD steht im Zentrum der Untersuchung. Vorausgesetzt wird, dass es das Menschenbild einer politischen Institution ist, in dem deren Werthaltung und das daraus resultierende Programm begründet sind. Da der gesellschaftliche Diskurs der Bundesrepublik seit 1949 das von Gleichheit und Würde geprägte Menschenbild konsensuell voraussetzt, ist hier das Kriterium vorgegeben zur Überprüfung politischer Programmatik. Die Leitfrage orientiert sich hier an der Ausdeutung des Eigenen und des Fremden.

\section{Inszenierung: Das Selbstbild der Partei}

Eine zentrale Strategie von Politikerinnen und Politikern ist die Selbstdarstellung. Ohne Selbstinszenierung und Imagearbeit ist kein politischer Auftritt denkbar.
Ein politischer Auftritt ist auch das Grundsatzprogramm einer Partei, mit dem diese sich ein Image schafft, eine Identität gibt. Zwei zentrale Aspekte werden nachfolgend als Phänomene der Imagearbeit vorgestellt, die im Grundsatzprogramm der AfD das Bild der Partei inszenieren.

\section{Demokratische Norm}

Das Programm der AfD beginnt mit der Präambel, deren erste Sätze lauten:

Wir sind Liberale und Konservative. Wir sind freie Bürger unseres Landes. Wir sind überzeugte Demokraten. (AfD 2016, S. 1)

Mit dieser Selbstidentifizierung teilt die AfD den Leserinnen und Lesern des Programms mit:

1) „Wir bewegen uns mit den Eigenschaften liberal und konservativ innerhalb des Rahmens, den die Norm des pluralistischen demokratischen Rechtsstaats vorgibt."

2) „Wir beanspruchen das in diesem Rechtsstaat verbürgte Recht der Freiheit."

3) „Wir sind als Demokraten legitime Vertreter dieses Rechtsstaats."

Die Botschaft dieser Selbstvorstellung lautet mithin: „Die AfD ist eine innerhalb der demokratischen Norm sich bewegende verfassungsgemäße Partei."

\section{DIE AFD STELLT SICH ALS ,PARTEI DES GESUNDEN MENSCHENVERSTANDES' DAR}

Weiteres Beispiel expliziter Selbstkonzeption ist die positiv konnotierte, bemerkenswerterweise in einfache Anführungszeichen gesetzte und damit eigentlich Distanz markierende Selbstzuschreibung, Partei des gesunden Menschenverstandes', ${ }^{4}$ die bedeutet:

4) „Die AfD ist eine Partei, deren Programm auf einem unverbildeten, natürlichen und sachbezogenen Urteil beruht, von der Bewertung des Gegebenen ausgeht und das praxisbezogen und konkret ist." 
In diesem Fall sagt die Selbstzuschreibung aus: „Die AfD ist eine von hohem, vor allem realitätsbezogenem Urteilsvermögen gekennzeichnete Partei." Übrigens: Ihren Wählern schreibt sie dieselbe Eigenschaft zu, wenn ausgeführt wird,

auf das politische Urteilsvermögen und die Verantwortungsbereitschaft der mündigen Bürger (AfD 2016, S. 5)

zu setzen. Hier wird eine Symbiose geschaffen.

Weiterhin stellt sich die AfD in die in der Bundesrepublik wohl beleumundete Tradition der sozialen Marktwirtschaft:

Als Partei der sozialen Marktwirtschaft will die AfD erreichen, dass [...]. (AfD 2016, S. 15)

Das bedeutet:

5) „Die AfD ist eine Partei, die diejenige Nachkriegsgeschichte der Bundesrepublik fortsetzt, die Konrad Adenauer und Ludwig Erhard begründet haben, und die Prosperität und Fortschritt erbracht haben."

Die Botschaft lautet: „In Zeiten der Globalisierung, die Unübersehbarkeit, Risiko und Unberechenbarkeit bedeutet, ist die AfD die Partei der wirtschaftlichen und sozialen Sicherheit und Verlässlichkeit."

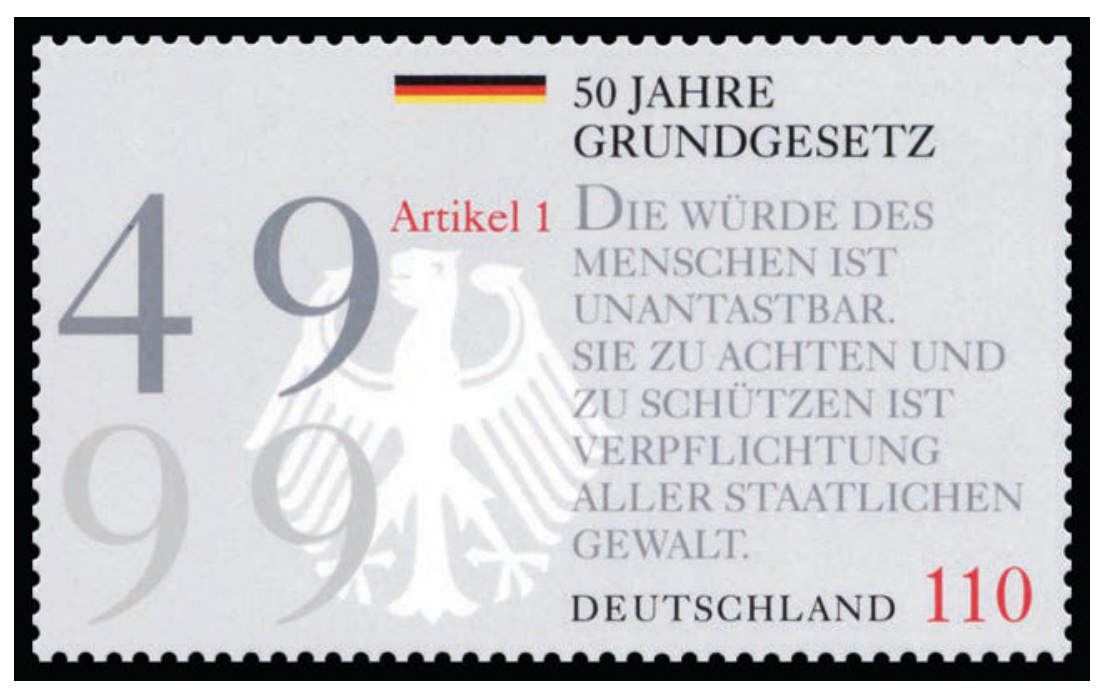

Wir sehen: Die AfD verwendet zu ihrer Selbstkonzeption stereotypisierende Elemente, die sie mit den Eigenschaften ,demokratisch', ,sachlich' und , verlässlich' versieht und damit mit solchen Eigenschaften, die gesellschaftlich konsensuell hohe Werte bezeichnen und die den Versuch darstellen, auf diesem Weg Zustimmungsbereitschaft hinsichtlich der politischen Ziele zu erzeugen.

\section{Aufräumer-Attitüde}

Abgesehen von diesen expliziten Selbstzuschreibungen ist natürlich die Formulierung von Haltungen und Einstellungen ein zentraler Identitätsmarker. Im Fall des AfD-Programms ist es die Markierung der „Aufräumer"-Partei, mit der die Autorinnen und Autoren sich und der Partei Identität schaffen. Diese wird als Politik- und Parteienkritik präsentiert. Die AfD inszeniert sich programmatisch als diejenige Partei, die alle vermeintlichen "Missstände“ in Politik und Gesellschaft beseitigt und Ordnung herstellt. Die Selbstbezeichnung Alternative drückt diesen Anspruch aus, ebenso wie die höchst frequente Verwendung entsprechender Leitwörter, Formeln und Sätze, die einen politischen Sachverhalt als Missstand, als überlebt, als nicht zeitgemäß bewertet.

\section{INSZENIERUNG DER AFD ALS \\ ,ALTERNATIVE' ORDNUNGSINSTANZ}

Leitwörter und Formeln dieser Politik- und Parteienkritik sind:

Parteibuchwirtschaft (AfD 2016, S. 5);

Ämterpatronage ist unter Strafe zu stellen (AfD 2016, S. 5);

Einführung eines Straftatbestandes der Steuerverschwendung (AfD 2016, S. 9);

Deutschlands Staatsapparat (AfD 2016, S. 3);

Die Abgeordneten unserer Parlamente haben ihre Funktion als Mandatare der Bürger verloren. Ihre Loyalität gilt zuerst der politischen Partei, der sie angehören. Von ihr erhalten sie ihre Wahlchancen und ihre Wahl sichert typischerweise ihren Lebensunterhalt. (AfD 2016, S. 6); 
Die politische Klasse Deutschlands hat das Wahlrecht und die Wahlverfahren im Laufe der Zeit immer perfekter ausgenutzt und angepasst, um den Einfluss des Volkes zu minimieren. (AfD 2016, S. 7)

Die sich fortsetzende Tendenz zum Berufspolitikertum hat der Monopolisierung der Macht Vorschub geleistet und die unübersehbare Kluft zwischen dem Volk und der sich herausgebildeten politischen Klasse vergrößert. Vetternwirtschaft, Filz, korruptionsfördernde Strukturen und verwerflicher Lobbyismus sind die Folge. (AfD 2016, S. 7)

Diese Attitüde wird in verschwörungstheoretischer Manier begründet:

Heimlicher Souverän ist eine kleine, machtvolle politische Führungsgruppe innerhalb der Parteien. Sie hat die Fehlentwicklungen der letzten Jahrzehnte zu verantworten. Es hat sich eine politische Klasse von Berufspolitikern herausgebildet, deren vordringliches Interesse ihrer Macht, ihrem Status und ihrem materiellen Wohlergehen gilt. Es handelt sich um ein politisches Kartell, das die Schalthebel der staatlichen Macht, soweit diese nicht an die EU übertragen worden ist, die gesamte politische Bildung und große Teile der Versorgung der Bevölkerung mit politischen Informationen in Händen hat. (AfD 2016, S. 3)

Politikerinnen und Politikern wird in diesem Text generell ein von Selbstinteresse geleitetes und nicht-reflektiertes, gleichgültiges Handeln unterstellt, womit sich eine Politik- und Demokratiefeindlichkeit ausdrückende Aggression manifestiert. Diese rechtspopulistische Politikkritik bringt die AfD stilistisch in denunziatorischer Diktion vor, die typische Sprachhandlung dieses denunziatorischen Stils ist die der Verleumdung und der Unterstellung, die grammatisch in apodiktischen, Widerspruch nicht zulassenden Aussagesätzen formuliert sind.

\section{PARALLELEN ZUM POLITISCHEN DISKURS DER RECHTEN PARTEIEN IN DER WEIMARER REPUBLIK}

Dieses Muster ist bekannt und hinsichtlich dieser partei- und politikkritischen Attitüde kann auf das historische Vorbild der Weimarer Republik verwiesen werden. Im politischen Diskurs der 1920er Jahre finden wir bei der extremen Rechten (NSDAP, DNVP) denselben diffamierenden und denunzierenden Stil, in dem über Politik, Politiker und Parteien geredet wird, dieselben apodiktischen Konstruktionen, dieselben Leitwörter wie z. B. im Programm der NSDAP von 1920:

Wir bekämpfen die korrumpierende Parlamentswirtschaft einer Stellenbesetzung nur nach Parteigesichtspunkten ohne Rücksicht auf Charakter und Fähigkeiten. (NSDAP 1920, S. 157)

In derselben Weise geißelt Hitler in einer Rede aus dem Jahr 1923 mit nationalistischer Argumentation das Parteiensystem:

So ist es gekommen, wie es kommen mußte, wie es immer wieder kommen wird, solange sich das deutsche Volk solche Regiererei der Parlamentsparteien gefallen läßt. [...] die Reichstagsmehrheit vertritt alles andere als nationale Interessen! Das Reich ist ja nicht mehr das deutsche Reich des deutschen Gefühls, sondern das Reich der Parteien! Diese aber kennen bloß ihre abgedroschenen Parteidogmen, ihre Parteigrundsätze, nicht aber deutsches Volksgefühl! (Hitler 1923, S. 181)

Wer aufräumt, bewegt sich in dem vergangenheitsorientierten Denkmuster der Restituierung. Dieses Denkmuster hat eine lexikalische Entsprechung in dem Wörtchen wieder. Es ist auffallend, wie häufig im Text des AfD-Programms beansprucht wird, etwas in einen ursprünglichen Zustand zurückversetzen zu wollen. Wieder wird in diesen Beispielen nicht in temporalrepetitiver Lesart, sondern im Sinn einer restituierenden Bedeutung verwendet:

Nationale Souveränität wiederherstellen (AfD 2016, S. 15);

Damit die Staaten Europas wieder zu Leuchttürmen für Freiheit und Demokratie in der Welt werden können (AfD 2016, S. 10);

Wir fordern, die Einbürgerung Krimineller zuverlässig zu verhindern, den Anspruch auf Einbürgerung abzuschaffen, den früheren Status Quo des Abstammungsprinzip (galt bis 2000) wieder einzuführen (AfD 2016, S. 18);

die militärischen Fähigkeiten der deutschen Streitkräfte wieder herzustellen, um Anschluss an die strategischen und operativen Erfordernisse zu finden. Diese von der AfD geforderte Wiederherstellung (AfD 2016, S. 21);

Wehrpflicht wieder einsetzen (AfD 2016, S. 22); 
Grundwehrdienst wieder einzusetzen (AfD 2016, S. 23);

Organe und Institutionen des Staates [müssen] sich wieder an das Recht halten (AfD 2016, S. 16);

Wiederherstellung unseres Rechtssystems (AfD 2016, S. 16); die AfD fordert Recht auf freie Rede für freie Bürger wieder ein (AfD 2016, S. 46);

Gleichberechtigung muss wieder Chancengleichheit bedeuten (AfD 2016, S. 44);

die Studienabschlüsse müssen wieder klare Inhalte vermitteln [...] Pädagogische Hochschulen [...] sind [...] wieder einzuführen (AfD 2016, S. 37);

dass an den Oberschulen wieder Bildungsinhalte im Mittelpunkt [...] stehen (AfD 2016, S. 39);

Das Abitur muss wieder eine Hochschulreifeprüfung werden (AfD 2016, S. 39);

dem Grundrecht zur freien Meinungsäußerung muss [...] wieder zur uneingeschränkten Geltung verholfen werden. (AfD 2016, S. 42)

\section{SPRACHLICHE MANIFESTATION DES VERGANGENHEITSORIENTIERTEN DENKENS: WIEDER}

Insbesondere in Bezug auf die Grundhaltung der AfD, die dem Autoritären als Prinzip verpflichtet ist und die eine Gesellschaft der Vertikalität idealisiert, kommt die vergangenheitsorientierte Restituierungshaltung zum Ausdruck, vor allem hinsichtlich der Rechtsprechung:

Dem Recht wieder zur Durchsetzung verhelfen (AfD 2016, S. 16);

Müssen sich die Organe wieder an das Recht halten (AfD 2016, S. 16);

Die Justiz muss wieder schneller arbeiten (AfD 2016, S. 16);

Respekt bei den jugendlichen Serientätern wiederherstellen. (AfD 2016, S. 16)

Wieder ist in diesen Beispielen mehrdeutig, was mit dem Wahrheitswert der Behauptung zu tun hat. Hinsichtlich des Wahrheitswertes der Aussage ist der Gebrauch dann unproblematisch, wenn in Bezug auf etwas nachweislich gegenwärtig nicht Existierendes (wie die allgemeine Wehrpflicht) gefordert wird, dieses einzuführen. Wieder bedeutet hier: Das Bezeichnete hat einmal existiert und soll erneut existieren. Strategisch suggestiv dagegen ist der Gebrauch von wieder, wenn eine Aussage mit der entsprechenden Forderung die Existenz eines Sachverhalts leugnet, die rein ideologisch motiviert ist: Wer fordert, das Rechtssystem wieder herstellen zu wollen, behauptet, dass das Rechtssystem gegenwärtig außer Kraft ist. Wer behauptet, dem Grundrecht der freien Meinungsäußerung wieder Geltung verschaffen zu wollen, behauptet, dieses Grundrecht sei gegenwärtig außer Kraft. Das ist diejenige sprachliche Camouflage-Strategie, mit der die AfD ein Klima des Misstrauens und der Konfrontation erzeugt, die die Ideologie des autoritären Ordnungsstaats zum Vorbild hat.

\section{DAS PROGRAMM DER AFD VERSTÖSST GEGEN POLITISCH KORREKTE SPRACHVORGABEN}

Zu dieser Strategie, die die Atmosphäre der Denunzierung und die Selbstidentität der Aufräumer-Partei erwirkt, zählt schließlich auch, was im Programm über political correctness $\mathrm{zu}$ lesen ist.

Politisch korrekte Sprachvorgaben lehnen wir ab (AfD 2016, S. 32),

lässt man apodiktisch verlauten und bewertet die Praxis als gesellschaftsschädigend:

Tatsachen sollen als solche benannt und nicht aus politischen Gründen verschleiert werden. [...] Was wahr ist, kann nicht „unkorrekt” sein. (AfD 2016, S. 33)

Argumentativ ausgestattet mit dem Wahrheitstopos, machen diese Sätze deutlich: Indem die Verfasserinnen und Verfasser des Textes die sprachlichen Regeln der political correctness als Verschleiern und - implizit als Lüge (,was wahr ist, kann nicht ...“) konzipieren, ignorieren sie, dass die Idee der political correctness ihren ethischen Ursprung in Empathie und Inklusion hat, indem diskriminierende Äußerungen (etwa bzgl. Geschlecht, Herkunft oder körperlicher Einschränkung) damit unterbunden werden sollen. Im Text dagegen wird das Image der Offenheit und Ehrlichkeit bedient, und wir werden im Verlauf sehen, dass diese Ablehnung politisch korrekter Sprache pro domo formuliert wurde: Das Programm der AfD zeichnet sich sprachlich durch Verstöße gegen diese sprachliche Ethik aus. 


\section{Ideologische Polysemie im AfD-Programm}

Einer der zentralen Gegenstände der Politolinguistik ist das Phänomen der ideologischen Polysemie (Dieckmann 1975, S. 70-75). Mit diesem Terminus wird die Eigenschaft von Wörtern bezeichnet, „die verschiedenen Ideologien gemeinsam sind und deren verschiedene Sinndeutungen nebeneinander in einer Sprache auftauchen“ (Dieckmann 1975, S. 71). Dieses Phänomen hat Josef Klein „Bedeutungskonkurrenz“ genannt: den bezeichnungsidentischen Gebrauch eines Schlagworts mit parteiabhängiger Spezifizierung der Bedeutung (Klein 1989, S. 22). ${ }^{5}$

Ideologische Polysemie entsteht, wenn ein politischer Akteur ein herausgehobenes, häufig verwendetes Wort des politischen Gegners, das dieser mit einer bestimmten Bedeutung versehen hat, mit einer neuen anderen Bedeutung versieht. Dies ist eine Strategie, die sich alle politischen Parteien zu eigen machen, so auch die AfD.

\section{WILLKOMMENSKULTUR UND GLEICHBERECHTIGUNG - IDEOLOGISCHE POLYSEMIE ALS STRATEGISCHES MITTEL}

Eines dieser Leitwörter des heutigen gesellschaftsbezogenen Diskurses ist der Ausdruck Willkommenskultur. Er wurde höchst frequent verwendet im Herbst und Winter 2015 / 2016, als Europa viele Geflüchtete aufgenommen hat und man - angesichts von Fremdenfeindlichkeit und xenophober Gewalt - dazu aufgerufen hat, die Geflüchteten willkommen zu heißen. Willkommenskultur war in diesem Kontext ein Leit- und Reizwort. Auch die AfD verwendet diese Bezeichnung - in gänzlich anderem Kontext und mit gänzlich anderer Bedeutung:

Die Alternative für Deutschland setzt sich für eine Willkommenskultur für Un- und Neugeborene ein. (AfD 2016, S. 31)

Willkommenskultur ist in diesem Zusammenhang die Bezeichnung für eine Haltung, die die Abtreibung verurteilt und für einen Anstieg der Geburtenrate plädiert. Die Brisanz dieser Verwendung ist noch insofern erhöht, als es sich in diesem Kontext um ein Plädoyer für die Geburt von Kindern von so gennanten „deutsch- stämmigen Frauen“ (dazu weiter unten) handelt, nicht von Kindern von Migrantinnen.

Zweites Beispiel ideologischer Polysemie ist die Neudeutung des Ausdrucks Gleichberechtigung. Im Grundsatzprogramm der AfD ist folgende Formulierung zu lesen:

Quotenregelungen [...] sind zu stoppen. Gleichberechtigung muss wieder Chancengleichheit bedeuten. [...] Die AfD unterstreicht [...] die grundrechtlich garantierte Gleichberechtigung von Mann und Frau (im Sinne von Chancengleichheit). Eine Gleichstellungspolitik im Sinne von Ergebnisgleichheit lehnt die AfD hingegen ab. (AfD 2016, S. 40f.)

Zwar wird die konventionelle Bedeutung, die im Frauenrechtsdiskurs die gleiche Ausstattung mit gesellschaftlichen und politischen Rechten von Frauen meint, bestätigt. Dafür ist Chancengleichheit ein Synonym. Gleichzeitig aber wird suggeriert, dass der Ausdruck Gleichberechtigung nicht als Synonym von Chancengleichheit verwendet wird, sondern so genannte Ergebnisgleichheit zum Ziel habe. Insofern diese Aussage im Kontext mit der Thematisierung von Quoten steht, die die AfD ablehnt, wird also Gleichberechtigung implizit im Sinn von Gleichstellungspolitik mit Ergebnisgleichheit im Sinn von Quotenregelungen gleichgesetzt.

In ihrem Grundsatzprogramm verwendet die AfD eine Reihe von Leitwörtern des politischen Diskurses, um sie in ihrem Sinn umzudeuten bzw. auf andere Referenzbereiche zu beziehen und neu- bzw. umzudeuten. Vornehmlich sind dies Leitwörter des Diversitätsdiskurses. Das Ziel ist klar: Dem politischen Gegner die Wörter zu nehmen, bedeutet, ihm seine Identität zu nehmen. Dieser Versuch wird im Fall der AfD nicht gelingen, denn das Evokationspotenzial von Wörtern wie Willkommenskultur und Gleichberechtigung ist zu festgelegt, um dieses Potenzial zu ändern. Als sprachliche Strategie des Grundsatzprogramms muss dieser Versuch aber als Befund der Analyse festgestellt werden.

\section{Das Menschenbild}

Politische Weltsichten sind entscheidend geprägt von einem je spezifischen Menschenbild, das Handlungspläne und Zukunftsentwürfe bestimmt. Politische Grundsatzprogramme reflektieren demnach Menschenbilder. 
Nach einem Menschenbild und dessen sprachlicher Repräsentierung fragen bedeutet, aus der Perspektive der linguistischen Anthropologie danach fragen, mit welchen sprachlichen Mitteln - lexikalisch-semantisch, funktional, argumentativ etc. - Menschen konstituiert werden. Die linguistische Anthropologie stellt die Frage nach dem „sprachgeprägte[n] Menschenbild“ (Hermanns 1994, S. 37) und setzt voraus: Menschenbilder verdichten sich in Stereotypen und sind Konstrukte in historischer Hinsicht und in Bezug auf die Perspektive der jeweiligen Akteure. ${ }^{6}$

\section{POLITISCHE ORIENTIERUNGEN MANIFESTIE- REN SICH IN SPRACHLICH GEFASSTEN MENSCHENBILDERN}

Stereotype sind in Sprache gefasste Vorurteile. Es sind Topoi, die oft

nur in Form von Attributen [erscheinen], die in Texten und Diskursen quasi leitmotivisch immer wieder einem Gegenstand bzw. einer Klasse zugeschrieben werden. (Hermanns 1994, S. 57)

Wir haben es dabei mit Emotion ausdrückenden Stereotypen bzw. Vorurteilen zu tun. Gefühle spielen eine höchst bedeutende Rolle bei der Entstehung von Menschenbildern überhaupt und erst recht im politischen, also argumentativ-strategischen, Kontext. ${ }^{8}$

Solche Topoi sind Komponenten einer sprachlichen Mentalitätsgeschichte, indem sie Haltungen und Ein-

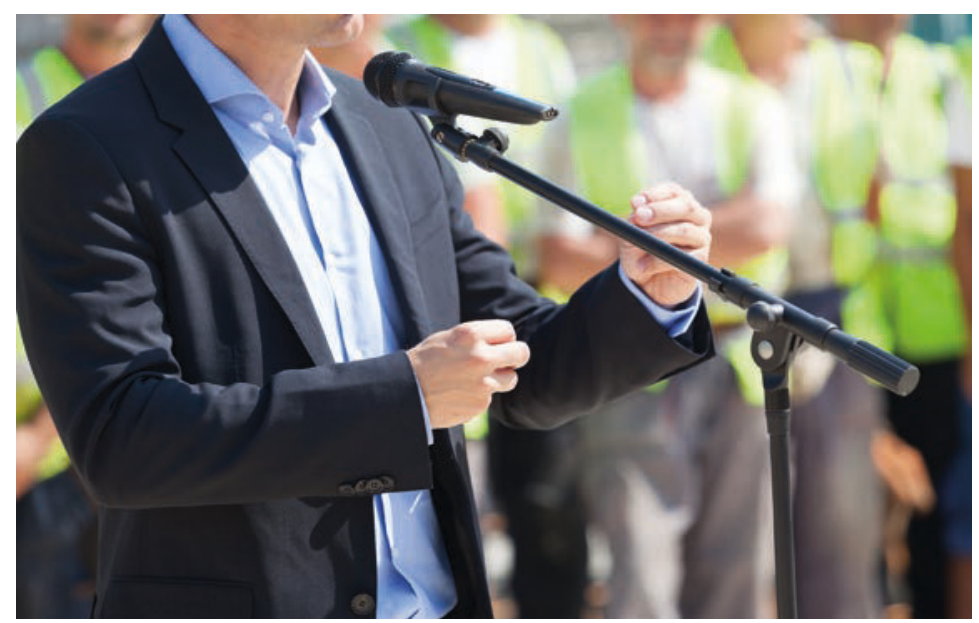

Ziele politischer Kommunikation: Überzeugen und Überreden stellungen ausdrücken, die ein gesamtgesellschaftliches Phänomen sein können, ein Phänomen einer bestimmten Epoche oder einer bestimmten gesellschaftlichen Gruppierung, wie etwa politischer Parteien. ${ }^{9}$

\section{ZIELE POLITISCHER KOMMUNIKATION: ÜBERZEUGEN UND ÜBERREDEN}

Im politischen Kontext Handelnde haben in einer Demokratie das primäre und prinzipielle Ziel, „Zustimmungsbereitschaften zu erzeugen“ (Lübbe 1975, S. 87). Unter dieser Voraussetzung fragt eine politisch informierte linguistische Anthropologie, also sozusagen eine politolinguistische Anthropologie, nach solchen Menschenbildern, die zu politischen, also persuasiven Zwecken erzeugt sind. Diese Menschenbilder lassen Rückschlüsse auf die Weltsicht z. B. von Parteien zu und machen damit ein Motiv ihrer politischen Orientierung erkennbar. Es geht damit um Menschenbilder, die im Kontext der Politik, also im Kontext der Strategie und der Persuasion, erzeugt sind und die die Basis und Begründung für eine bestimmte politische Argumentation bilden.

\section{DAS MENSCHENBILD DER AFD: DIE DISKUR- SIVE GRUNDFIGUR ,DAS EIGENE UND DAS FREMDE' ALS POLITISCHE LEITIDEE}

Politisches Handeln und Sprechen ist interessegeleitet, daher setzt die Kommunikation auf Überzeugen (argumentativ-rational) und Überreden (gefühlsmäßig-rhetorisch) (vgl. Burkhard 2008), daher ist die (partei)politische Weltsicht prinzipiell binär: Die eigene Partei wird aufgewertet, die des politischen Gegners abgewertet, die eigene Position wird als gut bewertet, die des politischen Gegners als schlecht, der von der eigenen Partei herbeigeführte Sachverhalt ist sinnvoll, nützlich und erwünscht, der vom politischen Gegner herbeigeführte ist sinnlos, unnütz und nicht erwünscht. ${ }^{10}$ Dies sind Beispiele für das Muster binären politischen Redens - dass von dieser Regel gelegentlich abgewichen wird und die Konsens- und Schnittstellensuche das politische Handeln und Reden bestimmen, ändert nichts an dem Prinzip der Binarität. 
Wenn dieses binäre Muster das Reden über Menschen prägt, dann wird nach dem Eigenen und dem Fremden gefragt. „Das Eigene und das Fremde“ stellt ein Begriffspaar dar wie Hellenen / Barbaren, Christen / Nicht-Christen, Weiße / Farbige, Arier / Juden, Deutsche / Ausländer. ${ }^{11}$ ,Das Eigene und das Fremde' ist eine diskursive Grundfigur (Busse 1997) und auf dem letztgenannten Begriffspaar beruht ja ganz eigentlich die Vorstellung von eigen und fremd. Wozu dienen Klassifizierungen wie diese? Im politischen Kontext sind sie Handlungsgrundlage. Die einen erfahren ein anderes - exkludierendes - oder gerade nicht ein anderes - inkludierendes - politisches Handeln als die andern. Auf jeden Fall ist der Unterschied das Handlungsmotiv: Weiße haben mehr Rechte als Farbige / Farbige haben dieselben Rechte wie Weiße.

Mit anderen Worten: ,Das Eigene und das Fremde' als politische Leitidee ist eine Angelegenheit einer auf diesem binären Menschenbild beruhenden Vorstellung politischen Handelns. Da diese Unterscheidung jedoch nicht einer sachlich-neutralen Feststellung entspricht, sind Klassifizierungen von Menschen im politischen Kontext immer auch wertend - entweder im Sinn der Feststellung von Gleichwertigkeit (die zwar dem Menschenrechtsartikel 1 entspricht, die aber Ungleichwertigkeit voraussetzt) oder im Sinn der Feststellung von Ungleichwertigkeit. Dann wird nicht nur danach gefragt, inwiefern sich die Angehörigen der eigenen Gruppe von denjenigen der anderen Gruppe unterscheiden. Sondern dann wird das Eigene und das Fremde von Menschen zur Erreichung bestimmter politischer Ziele auf- bzw. abgewertet, bestimmte Eigenschaften und ethnische Zugehörigkeit werden in einem Kausalzusammenhang gebraucht und Menschenbilder geraten zur Legitimationsgrundlage, auf der die Gesellschaft wertend abgebildet wird, aus der politische Forderungen abgeleitet werden und auf der Handlungsanforderungen formuliert werden. Damit ist eine rassistische Position beschrieben.

Diese Leitidee steht im Widerspruch zu Artikel 1 der Allgemeinen Erklärung der Menschenrechte, dessen erster Satz lautet:

Alle Menschen sind frei und gleich an Würde und Rechten geboren.

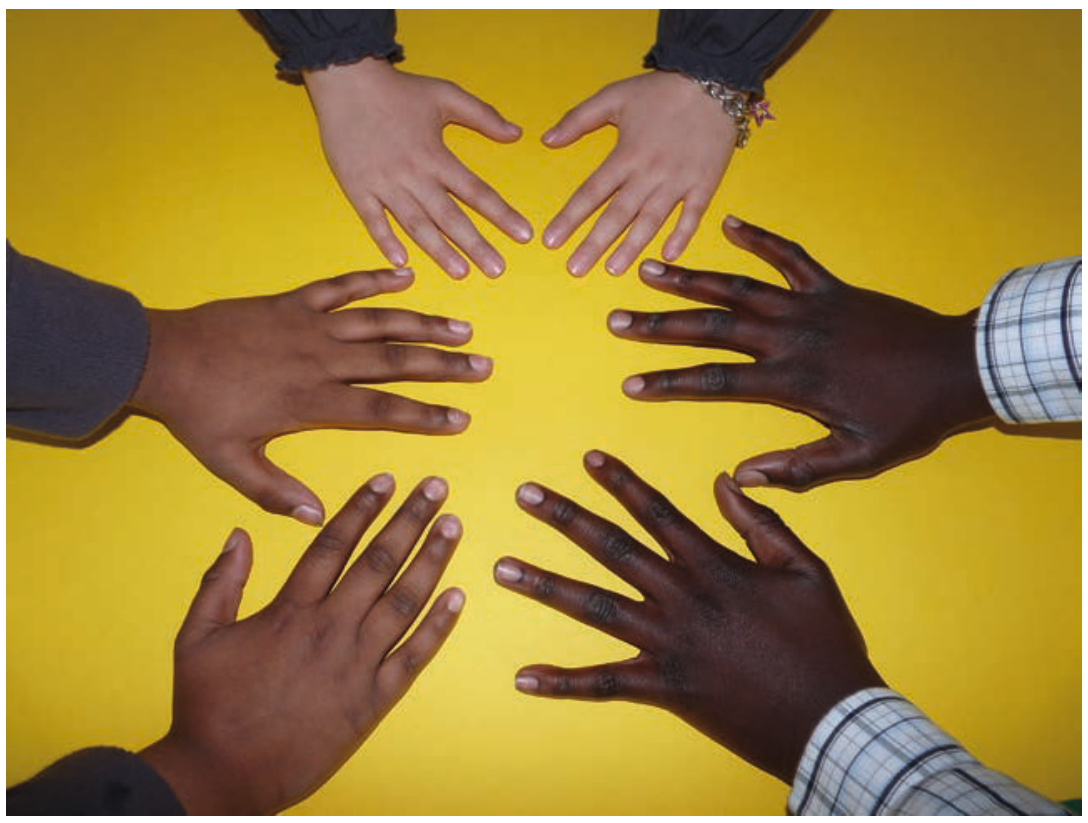

Die Unterscheidung in ,Das Eigene und das Fremde ${ }^{\prime}$ im politischen Kontext ist immer auch wertend.

Wer also Menschen in abwertender Absicht klassifiziert, um sich so entsprechenden Handlungsspielraum zu schaffen, spricht ihnen das Menschenrecht der gleichen Würde und Rechte ab. Gleichzeitig bewirkt diese Klassifizierung in „wert" und „unwert" eine Er- und Überhöhung dessen, was als „Eigenes“ gilt.

\section{DIE HERABSTUFUNG DES „FREMDEN“ BEWIRKT EINE ERHÖHUNG DES, EIGENEN“}

Auf der Grundlage dieser kulturlinguistischen Prämissen lauten die Ausgangsfragen: Mit welchen Zuschreibungen erstellt die AfD ihr Menschenbild? Welche politischen Forderungen leitet die AfD aus diesem Menschenbild ab? Wie begründet sie diese Forderungen?

Dem Menschenbild des Programms liegt, abgeleitet aus der diskursiven Grundfigur, das Eigene und das Fremde', das mentale Konzept ,Fremde sind ein Problem, Deutsche sind vor diesem Problem zu schützen' oder auch, konfrontativ ausgedrückt: ,Ausländer sind Feinde, die dem deutschen Volk Schaden zufügen, daher unerwünscht' zugrunde. Damit ist das Emotionalisierungspotenzial des Textes bezeichnet, der nicht nur über politische Positionen der AfD informieren will. Insofern politische Programme immer auch die Funktion haben, Haltungen und Einstellungen zu beeinflussen, ist der entsprechende beabsichtigte Effekt des AfD-Programms auch, eine emotionalisierte, in diesem Fall ablehnende, Einstellung der Leserinnen und Leser zu dem bezeichneten, durch das Menschenbild repräsentierten Sachverhalt zu erzeugen. 


\section{Das Eigene}

Mit der Feststellung „Derzeit gibt es über deutsches nationales Selbstbewußtsein nichts Erwähnenswertes zu vermelden" beschließt Fritz Hermanns 1995 seinen Aufsatz über die Neudeutungen, die deutsch, Deutsche/r und Deutschland nach der so genannten Wiedervereinigung erfahren haben (Hermanns 1995, S. 308). Das hat sich geändert. Während vor dem Aufkommen von AfD und Pegida Nationalismus kaum als ein weiter verbreitetes gesellschaftliches Phänomen anzusehen war, ist er mit Erscheinen dieser politischen Richtung in der Öffentlichkeit wieder deutlich bemerkbar geworden. Es überrascht deshalb nicht, dass im Programm der AfD deutsch und Deutschland Leitwörter zur Differenz markierenden Kennzeichnung des Eigenen sind.

\section{DER AUSDRUCK DEUTSCH ALS WERT-, DIFFERENZ- UND AUSSCHLUSSBEGRIFF}

Deutsch (bzw. die von der AfD dafür verwendeten Ausdrucksalternativen unser und einheimisch) haben limitische Funktion, das Eigene wird als ethnisch motiviert konzipiert und seine sprachliche Realisierung lässt erkennen: Ethnizität hat bei der AfD hohe Relevanz. ${ }^{12}$ Und: Ethnizität ist ein Konstrukt, ein sozial konstruierter Wissensbestand (Müller 2007, S. 512). ${ }^{13}$

Die linguistische Fragestellung lautet: In welchen Satzkontexten werden deutsch / Deutsche(r) / Deutschland bzw. die Bezeichnungsalternativen unser und einheimisch verwendet und was wird in diesen Kontexten ausgesagt?

\section{Biologischer Nationalismus}

Die Ethnie als solche wird bezeichnet mit Kollokationen wie deutsches Volk oder deutschstämmige Frau. Deutsch erhält hier einen Wert, es wird nicht als Herkunfts-, sondern als Bezeichnung mit wertender limitischer Funktion verwendet: Die erwünschte, zu schützende Eigenschaft deutsch grenzt von der unerwünschten Eigenschaft nicht-deutsch ab, ein Umstand, der im Kontext des Programms von größter Bedeutung ist, wie wir noch sehen werden. Und: deutsch in der
Verbindung deutsches Volk ist eine Eigenschaft, der Aufmerksamkeit zuteil werden soll - Wohl des deutschen Volkes ist eine rekurrente Formel. ${ }^{14}$ Die Entsprechungen mit Verwendung der Landesbezeichnung lauten: für Deutschland oder Reformen zum Wohle Deutschlands und, in expliziter Ausführung der Nützlichkeitsidee:

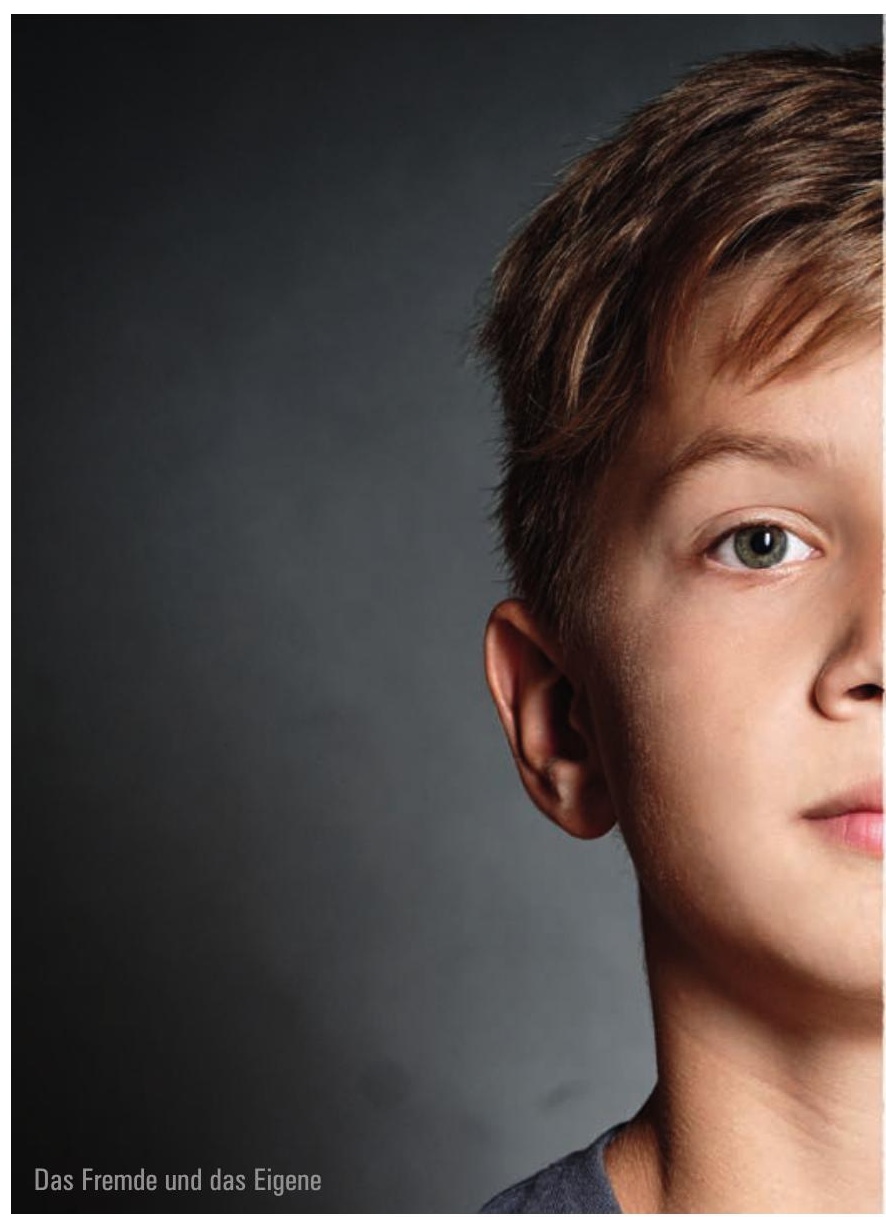

Für den Arbeitsmarkt qualifizierte Einwanderer mit hoher Integrationsbereitschaft sind uns willkommen [...] Davon strikt zu trennen ist die ungeregelte Asylzuwanderung, die dem Wirtschaftsstandort Deutschland nicht nutzt und der Gesellschaft schadet. (AfD 2016, S. 46) 
und deren als bedingungslos behauptete Vergabe wird mit Empörungsbereitschaft konstatiert:

Kinder bekommen unter bestimmten Bedingungen automatisch die deutsche Staatsangehörigkeit, auch wenn kein Elternteil Deutscher ist. (AfD 2016, S. 49) ${ }^{15}$

\section{VERWENDUNG DES AUSDRUCKS DEUTSCH - PARALLELEN ZUM PROGRAMM DER NSDAP 1920}

Auch hinsichtlich der Versprachlichung dieser nationalistischen Weltsicht lassen sich historische Bezüge markieren. Unübersehbar sind auch in diesem Fall des AfD-Programms Parallelen zum völkisch-nationalistischen Denken, das den Diskurs in der Weimarer Zeit bestimmt hat. Im Zusammenhang mit der Eigenschaft deutsch lässt sich das Programm der NSDAP aus dem Jahr 1920 parallelisieren:

Staatsbürger kann nur sein, wer Volksgenosse ist. Volksgenosse kann nur sein, wer deutschen Blutes ist, ohne Rücksichtnahme auf Konfession. [...] Wer nicht Staatsbürger ist, soll nur als Gast in Deutschland leben können und muß unter Fremdengesetzgebung stehen. [...] Wir fordern, daß sich der Staat verpflichtet, in erster Linie für die Erwerbs- und Lebensmöglichkeit der Staatsbürger zu sorgen. Wenn es nicht möglich ist, die Gesamtbevölkerung des Staates zu ernähren, so sind die Angehörigen fremder Nationen (Nicht-Staatsbürger) aus dem Reiche auszuweisen. [...] Jede weitere Einwanderung Nichtdeutscher ist zu verhindern. (NSDAP 1920, S. 34)

Insofern hier „biologistische [...] Formen des Nationalismus“ zum Ausdruck kommen, „welche die Nation selber als eine natürlich-rassische Einheit zu begründen" suchen (Geulen 2004, S. 442), sind die Parallelen zum Programm der NSDAP evident: In beiden Programmen wird der eigene Staat biologistisch-nationalistisch überhöht, in beiden Programmen sind Fremde, also Ausländer, als biologisch Nicht-Zugehörige ausgegrenzt.

Eine historische Parallele stellt ebenfalls die konstruierte Wirklichkeit der Deutschnationalen Volkspartei dar, in deren Programm aus dem Jahr 1920 zu lesen ist:
Wir fordern eine starke und stetige, nur durch deutsche Gesichtspunkte bestimmte auswärtige Politik, eine würdige, feste und geschickte Vertretung der deutschen Interessen und die Nutzbarmachung unserer wirtschaftlichen Kraft für die außenpolitischen Ziele Deutschlands. Der auswärtige Dienst ist allein nach Befähigung, Vorbildung und zuverlässiger deutscher Gesinnung zu besetzen. (DNVP 1920, S. 121)

Hier stellen wir dasselbe nationale Nützlichkeitsdenken fest, hier erkennen wir dieselbe Erhöhung der Eigenschaft deutsch, wie im Grundsatzprogramm der AfD.

\section{POSITIV-DEONTISCHE BEDEUTUNG DES AUSDRUCKS DEUTSCH: ZU SCHÜTZENDE EIGENSCHAFTEN WERDEN ALS DEUTSCH BEZEICHNET}

\section{Deutsch - ein kultureller Wert an sich}

Die zweite Gruppe bilden solche Formulierungen, in denen deutsch auf Kulturspezifika referiert:

deutsche Schriftsteller, deutsche Musiker, deutsche Designer (AfD 2016, S. 32);

Digitalisierung der Deutschen Literatur [...] eine von Deutschland zu leistende Aufgabe. Nur die eigene Bevölkerung und deutsche Literaturfachleute können deutsche Literaturwerke gewinnen (AfD 2016, S. 58);

Die Alternative für Deutschland bekennt sich zur deutschen Leitkultur (AfD 2016, S. 35);

die deutsche kulturelle Identität selbstbewusst verteidigen (AfD 2016, S. 32);

eine Islamkunde in deutscher Sprache für alle moslemischen Schüler, die in einen Ethikunterricht integriert wird. (AfD 2016, S. 40)

In Verbindungen wie diesen wird nicht nur denotativdeskriptiv auf Sachverhalte Bezug genommen, sondern deutsch ist in solchen Verbindungen Legitimationsvokabel, weil sie nicht nur eine Herkunft bezeichnet, sondern eine Eigenschaft bewertet. Der mit deutsch bezeichneten Eigenschaft wird, um den Terminus Fritz Hermanns' zu verwenden, ${ }^{16}$ eine positiv-deontische Bedeutung zugeschrieben, also eine Bedeutung mit ei- 
nem Handlungsappell, der in dem Kontext, in dem der Ausdruck deutsch im Parteiprogramm der AfD vorkommt, bedeutet: 'unbedingt zu erhalten'. ${ }^{17}$ Voraussetzung für diesen Appell ist das in dem Programm konstruierte Szenario einer Masseneinwanderung von Migranten und Flüchtlingen, die die deutsche Kultur in Gefahr bringt. In diesem Szenario stellt sich die Eigenschaft deutsch als Wert an sich dar. Die deontische Bedeutung von deutsch tritt insbesondere in der Kollokation deutsche Leitkultur und ihrer Definition zutage:

Die Alternative für Deutschland bekennt sich zur deutschen Leitkultur, die sich im Wesentlichen aus drei Quellen speist: erstens der religiösen Überlieferung des Christentums, zweitens der wissenschaftlich-humanistischen Tradition, deren antike Wurzeln in Renaissance und Aufklärung erneuert wurden, und drittens dem römischen Recht, auf dem unser Rechtsstaat fußt. (AfD 2016, S. 35)

Der Subtext unter dieser Erklärung lautet: Wer nicht in dieser Geschichte wurzelt, ist ausgeschlossen. Die Eigenschaftszuschreibung deutsch hat hier die Funktion einer Differenzchiffre, die den Unterschied zwischen ,wertvoll' und ,wertlos' markiert. Deutsch - um den Gebrauch im Programmtext zusammenzufassen, wird also nicht im Sinn einer Herkunftsbezeichnung, sondern im Sinn einer hoch bewerteten „ethische[n] Bestimmung" verwendet (Loewenstein 2001, S. 291) unter der Prämisse eines drohenden Verlusts.

\section{DIE KOLLOKATION DEUTSCHE LEITKULTUR WIRKT ALS DIFFERENZCHIFFRE, DIE DIE UNTERSCHEIDUNG ZWISCHEN ,WERTVOLL' UND ,WERTLOS' BETONT}

Eine weitere limitische Vokabel mit derselben Funktion ist unser. Sie wird synonym zu deutsch verwendet und reklamiert als solches Besitz oder Qualität:

Wiederherstellung unseres Rechtssystems (AfD 2016, S. 16); die deutsche Staatsangehörigkeit untrennbar mit unserer Kultur und Sprache verbunden (AfD 2016, S. 49);

dem römischen Recht, auf dem unser Rechtsstaat fußt (AfD 2016, S. 35);

Der hohe Standard unseres Bildungssystems (AfD 2016, S. 47);
Die Einhaltung des Rechts [...] ist Voraussetzung für unsere Freiheit. (AfD 2016, S. 16)

Insofern diese als Realisat von ,das Eigene' ebenfalls die Funktion eines Ausschlusskriteriums hat, wird mit dem Gebrauch von unser diese Diskriminierungsfunktion in höchstem Maße evident:

Ein orthodoxer Islam, der unsere Rechtsordnung nicht respektiert [...] ist mit unserer Rechtsordnung und Kultur unvereinbar. (AfD 2016, S. 34);

Eine orthodoxe Auslegung des Islam ist mit unserer freiheitlich-demokratischen Grundordnung nicht vereinbar. (AfD 2016, S. 40)

Was geschieht hier sprachlich? Unser stiftet eine Gemeinschaft, benennt eine Zugehörigkeit und grenzt damit alles aus, von dem erklärt wird, dass es bestimmten Aspekten dieser Gemeinschaft, wie hier der Rechtsordnung, nicht entspricht. Das ist hinsichtlich der sprachlichen Ausführung insofern bemerkenswert, als hier eine sprachliche Implikatur ihre Wirkung entfaltet: Die verdeckte implizierte Aussage lautet, dass es einen die deutsche Rechtsordnung nicht akzeptierenden Islam gibt. Dieser Sachverhalt aber ist erstens wenig existent, zweitens bereits in der geltenden Rechtsprechung vorgesehen. Abgesehen davon bestätigt der Inhalt nicht nur das Denken der nationalkonservativen Orientierung, sondern ist allgemeiner gesellschaftlicher Konsens. Weil aber im Kontext dieses Parteiprogramms weder relativiert noch auf die bestehende Gesetzeslage verwiesen wird, gerät diese Feststellung zu einer Form von Diffamierung und Denunzierung.

\section{DAS PRONOMEN ,UNSER' IMPLIZIERT SPRACHLICH DIE AUSGRENZUNG ANDERER VORSTELLUNGEN UND ANSICHTEN}

Exkludierende Wirkung hat auch ein Satz wie der folgende, der nur an der Oberfläche das Gegenteil von Ausschluss aus der Gemeinschaft bedeutet:

Viele Muslime leben rechtstreu sowie integriert und sind akzeptierte Mitglieder unserer Gesellschaft. (AfD 2016, S. 34)

Indem auf Muslime mit den Zuschreibungen rechtstreu und integriert Bezug genommen wird, werden sie positiv diskriminiert, und indem sie ausdrücklich zu Mit- 
gliedern unserer Gesellschaft, also zu Mitgliedern einer Gesellschaft, die eben nicht ihre Gesellschaft ist, erklärt werden, werden sie als eigentlich nicht dazugehörig sprachlich ausgegrenzt.

Auf den Gebrauch von einheimisch in derselben Funktion ist ebenfalls hinzuweisen:

Die Erschließung der einheimischen Potenziale (AfD 2016, S. 50)

wird gefordert, um damit nicht-einheimische, also nicht-deutsche, Potenziale auszuschließen und abzuwerten, und in explizitester und die entsprechende Werthaltung nicht verbergender Form wird die Ideologie des Multikulturalismus verurteilt:

Die Ideologie des Multikulturalismus, die importierte kulturelle Strömungen auf geschichtsblinde Weise der einheimischen Kultur gleichstellt und deren Werte damit zutiefst relativiert, betrachtet die AfD als ernste Bedrohung für den sozialen Frieden und für den Fortbestand der Nation als kulturelle Einheit. (AfD 2016, S. 32) ${ }^{18}$

Die Eindeutigkeit dieser Aussage lässt weder sprachlich noch sachlich zu wünschen übrig: Hohe deutsche Kultur ist nicht gleichzustellen mit niederer nichtdeutscher Kultur. ${ }^{19}$

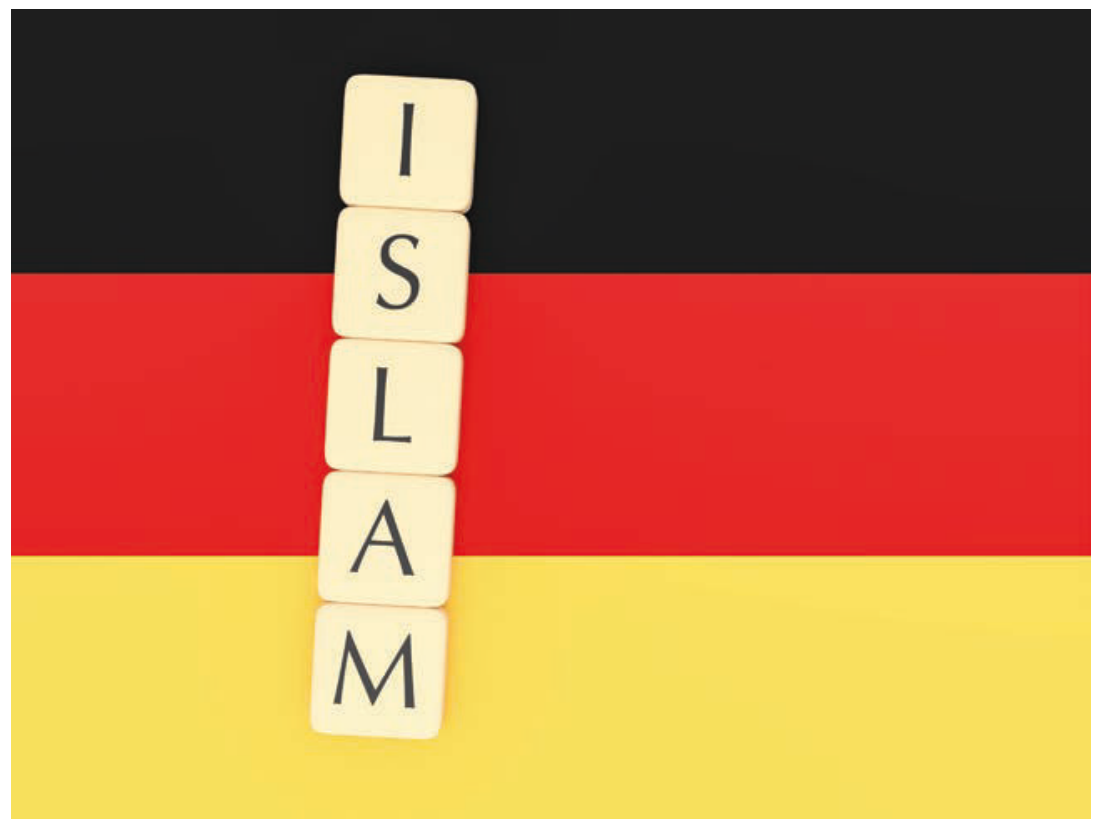

Islam in Deutschland
Was deutlich wird im Zuge der exkludierenden Argumentation der AfD: Das AfD-Programm beruht auf einer biologistischen, kulturalistisch-nationalistischen Weltanschauung mit gegen ,Nicht-Zugehörige‘ gerichteten Ungleichheitsvorstellungen und bedient nationalistische Ressentiments, die historisch aus dem völkisch-nationalistischen Geist des frühen 20. Jahrhunderts ableitbar sind (der wiederum im rassistisch-nationalistischen Denken des späten 19. Jahrhunderts seine Wurzeln hat, auf die hier nicht eingegangen werden kann).

\section{NATIONALISTISCHE RESSENTIMENTS, DIE HISTORISCH AUS DEM VÖLKISCH- NATIONALISTISCHEN GEIST DES FRÜHEN}

\section{JAHRHUNDERTS ABLEITBAR SIND}

Deutsch (und die beschriebenen Ausdrucksalternativen) ist im Kontext des Grundsatzprogramms kein Herkunfts-, sondern ein Wert-, Differenz- und Ausschlussbegriff, dessen Verwendung deutlich macht, dass die im Programm der AfD imaginierte Gemeinschaft (nach Anderson, „imagined communities“) der Deutschen die geschlossene Gesellschaft der Deutschstämmigen darstellt. Die Identifikationsinstanz des Nationalismus AfD'scher Provenienz ist damit nicht die des Staates, sondern die des Volkes. ${ }^{20}$ Die Deutschen (siehe deutschstämmige Frau) sind in der Lesart dieses Volksnationalismus die Träger der Nation, nicht die Bürgerschaft.

\section{Das Fremde}

Gegenwort $\mathrm{zu}$,das Eigene' ist ,das Fremde' und bezeichnet das Nichtzugehörige, Andere, dem dann Relevanz zugeschrieben wird, wenn ethnozentrisch argumentiert wird. Während ,das Eigene' das ist, dessen man sich vergewissert, das mit Zutrauen und Vertraut- 
heit korrespondiert, konzipiert man ,das Fremde' als das Abweichende. Im Grundsatzprogramm der AfD wird dieses Abweichende als religiöses Phänomen und als der gesellschaftlichen Norm widersprechendes Phänomen konzipiert.

\section{Antiislamismus}

Der Islam gehört nicht zu Deutschland. (AfD 2016, S. 34)

Das ist wohl die meist zitierte Aussage aus dem Programm der AfD, mit dem sie ihre fremdenfeindliche und Religionsfreiheit leugnende Haltung dokumentiert und die den Diskurs dynamisiert und polarisiert hat. Er wurzelt in der das Gegenteil bezeichnenden Feststellung Der Islam gehört zu Deutschland. In dieser Version begrüßt der damalige Bundesinnenminister Wolfgang Schäuble im Jahr 2006 die erste Islamkonferenz, der damalige Bundespräsident Christian Wulff wiederholt sie am 3. Oktober 2010 aus Anlass seiner Rede zu 20 Jahre deutsche Einheit, auch die Kanzlerin verwendet sie schließlich am 14. Januar 2015.

\section{DAS ,FREMDE“; „DER ISLAM GEHÖRT" FÜR DIE AFD „NICHT ZU DEUTSCHLAND“}

Dieser Satz ist mehrdeutig und verdichtet den kontroversen politischen Islam-Diskurs. Seine Mehrdeutigkeit ist verursacht von dem polysemen gehört, das zwei Lesarten zulässt. Als Feststellung eines historisch begründeten Sachverhalts bedeutet er:

Weil der Islam historisch-kulturell nicht in Deutschland verwurzelt ist, gehört er im Hinblick auf die geschichtliche Entwicklung nicht zu Deutschland.

Daraus leiten die Islamgegner dann ihre eigentliche Botschaft ab:

Der Islam und Moslems sind daher fremd, wir wollen sie daher hier nicht haben.

Was die AfD-Gegner und Befürworter religiöser Freiheit mit ihrer das Gegenteil behauptenden Version Der Islam gehört zu Deutschland dagegensetzen, lässt sich aus derjenigen Lesart von gehört ableiten, aus der diese Aussage resultiert:

Der Islam ist Realität in Deutschland, und als solche gehört er zu Deutschland.
Wir haben es hier also mit zwei Lesarten zu tun, einer, die den Ursprung, und einer, die die Zugehörigkeit bezeichnet.

Diese Realität ist Gegenstand des AfD-Programms, die die Verfasserinnen und Verfasser des Programms in hohem Maß relevant setzen, indem sie diese Realität in den Kontext von Kriminalität und fehlender Bildung stellen.

\section{Kriminalisierende Xenophobie}

Während das Eigene sich in der einen Identitätsvokabel deutsch verdichtet, hat das Fremde eine Reihe von Eigenschaften - immer aber solche Eigenschaften, die als außerhalb der gesellschaftlichen Norm konzipiert werden: Die Relevanzstruktur der AfD-Wirklichkeit besteht in der Konzeption von Nicht-Deutschen, die nach Deutschland kommen (als Einwanderer, um Asyl Bittende oder als Flüchtlinge ${ }^{21}$ ), dominant mit den Eigenschaften ,un-' bzw. ,schlecht gebildet', ,Sozialhilfeempfänger', ,kriminell', ,kinderreich':

Niedrigqualifizierte wandern überwiegend über missbräuchliche Asylanträge zu (AfD 2016, S. 51);

Mehrzahl der Täter im Bereich der organisierten Kriminalität sind Ausländer;

Erheblicher Anteil von Ausländern im Bereich der Gewaltund Drogenkriminalität (AfD 2016, S. 17);

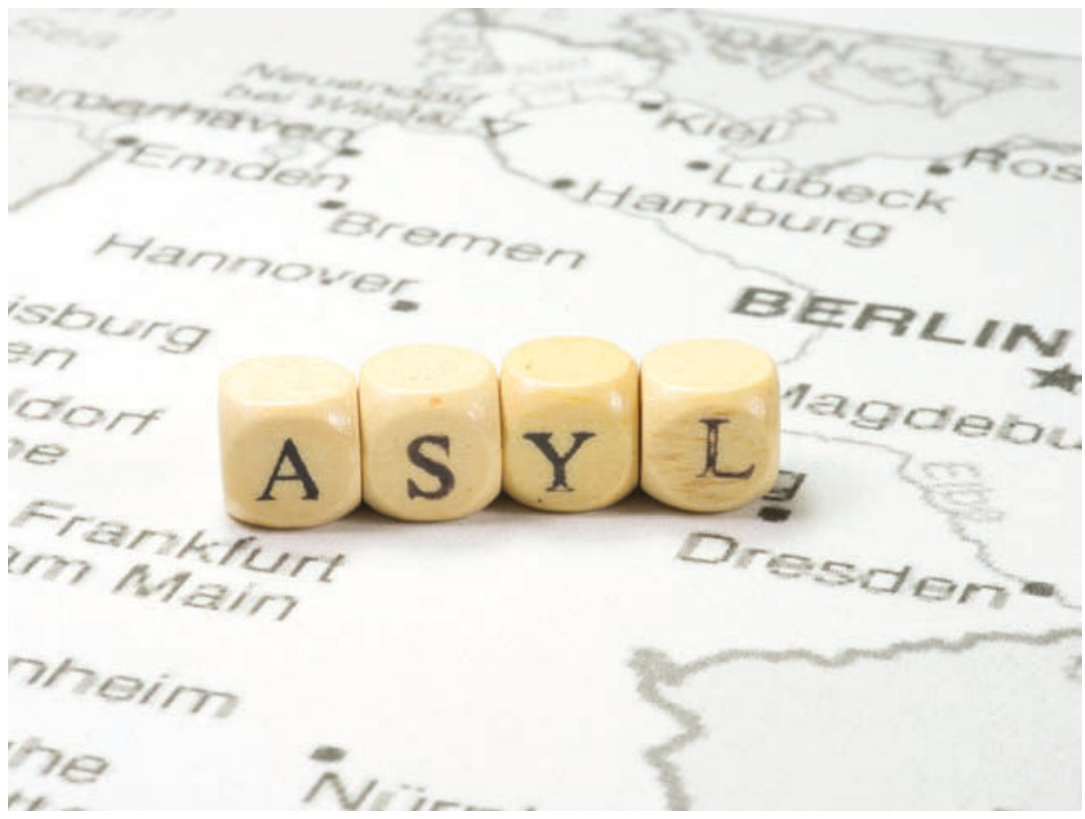

Asyl in Deutschland 
Einwanderung in die Sozialsysteme (AfD 2016, S. 42);

über das Asylrecht in das deutsche Sozialsystem einzuwandern (AfD 2016, S. 48);

Dabei hat sich in den vergangenen Jahren gezeigt, dass insbesondere muslimische Migranten in Deutschland nur ein unterdurchschnittliches Bildungs- und Beschäftigungsniveau erreichen. (AfD 2016, S. 28);

Einwandererkriminalität - nichts verschleiern, nichts verschweigen (AfD 2016, S. 48);

Enttäuschte Hoffnungen auf Wohlstand bergen die Gefahr, dass viele in die Kriminalität abgleiten. (AfD 2016, S. 48)

Die sprachliche Ausführung, mit der die Botschaft vermittelt wird, hat verschiedene Versionen. Wenn etwa negativ-abwertende Aussagen über Nicht-Deutsche gemacht werden, die der Diskriminierung und der Legitimierung entsprechender Forderungen dienen, sind sie entweder generalisierend (d.h. ohne spezifizierende Angaben), wie z. B. in Bezugnahmen auf Bildung und Beschäftigung, oder sie stellen eine überdurchschnittliche Quantität fest in Aussagen über Kriminalität.

\section{NEGATIV-ABWERTENDE AUSSAGEN ÜBER NICHT-DEUTSCHE: EINWANDERER- KRIMINALITÄT, MISSBRÄUCHLICHE ASYLANTRÄGE, UNTERDURCH- SCHNITTLICHES BILDUNGSNIVEAU}

Wenn hingegen Aussagen über Nicht-Deutsche auf einen positiven Sachverhalt referieren, haben sie konzessive Funktion und quantitative Angaben sind einschränkend:

Viele Muslime leben rechtstreu sowie integriert und sind akzeptierte Mitglieder unserer Gesellschaft. Die AfD verlangt jedoch zu verhindern, dass sich islamische Parallelgesellschaften mit Scharia-Richtern bilden [...]. (AfD 2016, S. 34)

Die Aussage dieser Sätze erzeugt die grammatische Konstruktion. Der erste Satz ist als Feststellung deshalb ein Zugeständnis, weil er durch das im zweiten Satz verwendete jedoch einen konzessiven, und damit zweitrangigen, Status erhält. Der eigentliche Fokus der Aussage und Träger der Botschaft ist der zweite Satz, indem die Chiffren islamische Parallelgesellschaften und Scharia-Richter die gewünschte Abwehr evozieren: Die AfD setzt hier auf das Angst erzeugende und abwehrende Evokationspotenzial dieser Chiffren und bewirkt damit die ablehnende Haltung, die sie für die Zustimmung zu ihrem Programm benötigt.

Mit Aussagen wie diesen werden zwei Strategien verfolgt: Sie dienen der Diskriminierung, die die Vorbereitung dafür darstellt, Zustimmung zu erzeugen und die Legitimierung entsprechender politischer Ziele zu schaffen. Diese werden z. B. im Zusammenhang mit der Feststellung einer „krisenhafte[n] Zuspitzung der demografischen Entwicklung" (AfD 2016, S. 29) formuliert. Insofern - vor dem Hintergrund der geschilderten Vorbereitung - das Argument des Zuzugs von Migranten als „Masseneinwanderung“ und als „kein geeignetes Mittel“ evaluiert wird, wird in der Logik dieser Aussage konsequent abgeleitet: „Mehr Kinder statt Masseneinwanderung" (AfD 2016, S. 27) - geboren von „deutschstämmigen Frauen“.

Auch hinsichtlich dieser Kommentierung einer aus biologistisch-nationalistischer Sicht besorgniserregend niedrigen Geburtenrate lassen sich historische Parallelen ziehen. Ebenfalls im Kontext eines Parteiprogramms wird das Thema in der frühen Weimarer Republik in derselben völkisch-rassistischen Diktion behandelt. Im Programm der Deutschnationalen Volkspartei von 1920 ist zu lesen:

Die breiten Volksmassen sind über die Gefahren des Geburtenrückganges aufzuklären; bei gesetzgeberischen Maßnahmen sind kinderreiche Familien grundsätzlich besonders zu berücksichtigen. (DNVP 1920, S. 123)

Dem im AfD-Programm vermittelten binären Menschenbild entspricht folgender Befund: Die Binarität des Programms entsteht durch die Polarisierung der als Wert an sich konzipierten Eigenschaft des mit deutsch bezeichneten Eigenen, das mit Ausschlussmotiven konfrontiert wird, die die Eigenschaften des Fremden begründen. Voraussetzung für die Konstellation dieses Denkmusters ist die Feststellung: Jeder Einwan- 
derer hat eine Bringschuld, sich zu integrieren (AfD 2016, S. 51). Sie inszeniert eine zu nichts verpflichtete überlegene Mehrheitsgesellschaft der Deutschen, der die mit Auflagen, Pflichten und Leistungen versehene Minderheit von Fremden gegenübergestellt wird. Was also ist Integration? Die AfD imaginiert nicht eine integrierte Gesellschaft, in der alle dieser Gesellschaft Zugehörigen ihren jeweiligen Integrationsbeitrag leisten, sondern eine Integration von fremden Minderheiten in die deutsche Mehrheitsgesellschaft. Integration wird damit nicht als eine gesamtgesellschaftliche Aufgabe verstanden, sondern als eine Leistung, die ausschließlich die zu Integrierenden zu erbringen haben.

\section{INSZENIERUNG DER, DEUTSCHEN MEHRHEITSGESELLSCHAFT' ALS ÜBERLEGENE GESELLSCHAFT GEGENÜBER DER ,FREMDEN' MINDERHEIT}

\begin{abstract}
Das Programm als Regelverstoß - ein Fazit
Menschliche Kommunikation funktioniert auf der Basis von Spielregeln, die eingehalten werden müssen, wenn die Kommunikation erfolgreich sein soll. Zu diesen Spielregeln zählt z. B. ein öffentliches Reden mit und über Menschen, das prinzipiell die Menschenwürde achtet, das die Menschenrechte respektiert, das wahr ist. Dieses Prinzip ist im Grundgesetz der Bundesrepublik normiert. Die Gesellschaft der Bundesrepublik ist eine auf den Werten des Grundgesetzes fundierte Gesellschaft, insofern sind die Grundrechte des Grundgesetzes die verpflichtende Wertegrundlage der Gesellschaft. Das Menschenbild des Grundgesetzes ist geprägt von dem Kennzeichen der Gleichheit und der Gerechtigkeit. Damit erhebt die Verfassung etwa die Gleichbehandlung von Menschen zum gesellschaftlichen und politischen Grundprinzip.
\end{abstract}

Diese im Grundgesetz normierten Werte des Menschenrechts haben einen unmittelbaren Bezug zum Zivilisationsbruch des Nationalsozialismus - der erste Satz des Grundgesetzes „Die Würde des Menschen ist unantastbar" spiegelt unmittelbar diese Erfahrung und

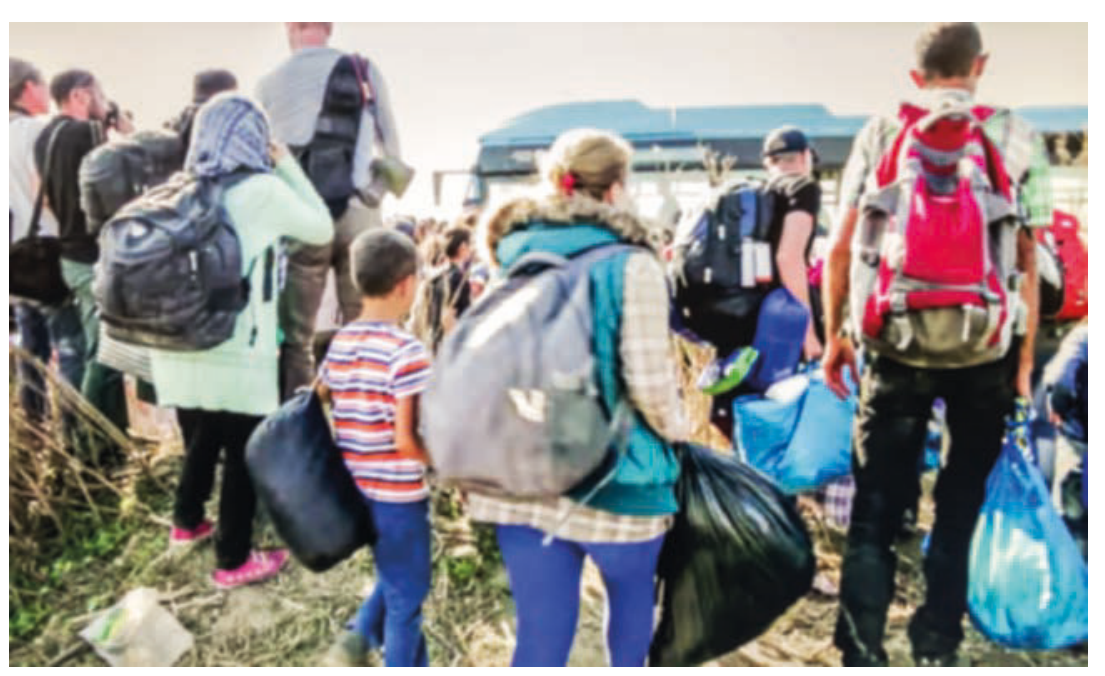

Asylsuchende in Deutschland

legt eine Haltung fest, die seither gesellschaftlicher Konsens ist. Diesen Konsens kündigt das AfD-Programm auf.

\section{SPRACHLICHE GEWALT: MENSCHEN WERDEN IN KATEGORIEN VON ,GUT“ UND ,SCHLECHT' EINGETEILT}

Diese Aufkündigung ist in einem weiteren Sinn als sprachliche Gewalt zu bewerten. Sprachliche Gewalt ist nicht nur in den bekannten Ausprägungen zu finden, die jegliches Tabu des Nichtsagbaren ignorieren und für die Beispiele ohne Zahl in den so gennanten sozialen Medien zu finden sind. Weitergefasst ist sprachliche Gewalt auch dann am Werk, wenn die Gleichheits- und Gerechtigkeitsprinzipien des Grundgesetzes missachtet werden, wenn Menschen in Kategorien von gut und schlecht einsortiert werden, wenn damit Bezüge zu nationalistisch-rassistischem, völkischem Denken nachweisbar sind, das nach der Erfahrung mit Nationalismus und Rassismus den Prinzipien des Grundgesetzes widerspricht. Sprachliche Gewalt bedeutet demnach auch, Rassismus auszudrücken und Menschen kollektiv ihre Gleichwertigkeit abzusprechen. Das ist ein Tabubruch, dessen kalkulierte Provokation in einer rechtspopulistisch-rassistischen Weltanschauung mit Ungleichheitsvorstellungen von ,Nicht-Zugehörigen' besteht und der xenophobe Ressentiments bedient, die historisch aus dem rassistischen Geist des frühen 20. Jahrhunderts ableitbar sind. Dieser Tabubruch verschiebt systematisch die Grenzen des Sagbaren.

Die AfD ist vom Bundesverfassungsgericht nicht verboten, und wie streng die Maßstäbe eines Parteiverbots sind, wissen wir aus dem Verbotsverfahren der NPD. Gefährdung der freiheitlich-demokratischen Grundordnung im Sinn ihrer aggressiven Bekämpfung mit dem Ziel ihrer Beseitigung und damit Verfassungswid- 
rigkeit ist das verfassungsrechtliche Ausschlusskriterium. Dieser Sachverhalt ist im Fall der AfD wohl generell nicht gegeben. ${ }^{22}$

\section{DAS SPRACHLICH MANIFESTIERTE MENSCHENBILD DER AFD VERSTÖSST GEGEN DIE DEMOKRATISCHEN WERTE}

Muss aber deshalb die AfD als Partei des äußeren rechten Randes unwidersprochen akzeptiert werden? Muss die Demokratie sie sich leisten und die Verbreitung menschenfeindlicher Grundsätze, die als gegen die Werte des Grundgesetzes gerichtet lesbar sind, hinnehmen? Eine Partei und ihr Programm gehört als Gegenstand des politischen Diskurses in die Öffentlichkeit einer demokratischen, pluralistischen Gesellschaft und muss auch der kritischen Bewertung unterzogen werden. Wenn diese Bewertung den Befund zutage fördert, dass hier diskriminierend und nationalistisch argumentiert wird, ist es Aufgabe des Diskurses, etwaige Normalisierungseffekte zu verhindern und als Korrektiv zu agieren. Die Politolinguistik kann hier einen wichtigen Beitrag leisten.

Deutlich machen, warum die AfD nicht eine Partei ist "wie jede andere“, sondern eine Partei, die dem demokratischen Wertesystem nicht entspricht (obwohl sie verfassungsrechtlich nicht verboten ist), ist auch eine Aufgabe der politischen Linguistik. Sie kann den Rechtspopulismus der AfD (sprach)analytisch abgeleitet als das darstellen, was er ist: im Widerspruch mit den demokratischen Werten der sozialen Gerechtigkeit und des gesellschaftlichen Ausgleichs stehend, ein Menschenbild dokumentierend, das nicht mit dem Grundgesetz vereinbar ist, weil es nicht von der Gleichheit der Menschen ausgeht; eine vertikalisierte Gesellschaft bedienend, die nicht eine Gesellschaft des sozialen Ausgleichs und der Chancengerechtigkeit ist, sondern eine hierarchisierte Gesellschaft von Klassen und Kompetenzen mit unterschiedlicher Wertigkeit.

\section{Anmerkungen}

1 Das Phänomen hat die Politolinguistik als generelle politiksprachliche Erscheinung bereits benannt: „Den Eindruck zu erwecken, man habe sich klar und eindeutig geäußert, und damit zu kaschieren, dass man systematisch weite Deutungsspielräume und / oder alternative Lesarten eröffnet hat, ist ein Strategietypus, der vor allem verwendet wird, um Adressatengruppen mit divergierenden Präferenzen gleichzeitig zu bedienen, um sich Operationsspielräume offen zu halten und um der eigenen Äußerung im Bedarfsfall eine andere Interpretation als die zunächst nahe liegende geben zu können." (Klein 1998, S. 386)

2 AfD und Bündnis 90 / Die Grünen treffen sich z. B. in der Forderung der AfD „Verbraucherschutz modernisieren und stärken“ (AfD 2016, S. 54). Die Auslassungen zum Klimawandel entbehren dagegen jeglicher wissenschaftlicher Basis und sind nachweislich falsch (vgl. AfD 2016, S. 61). Mit der SPD gehen zusammen z. B. die Forderung eines einheitlichen gesetzlichen Mindestlohns, die der Aufwertung des ländlichen Raums oder des verstärkten Tierschutzes.

3 Zum Godesberger Programm vgl. Hermanns (1989).

4 Als ,Partei des gesunden Menschenverstandes' setzen wir auf das politische Urteilsvermögen und die Verantwortungsbereitschaft der mündigen Bürger (AfD 2016, S. 5).

5 Das Gegenstück ist die so genannte „Bezeichnungskonkurrenz", die sich auf den referenzidentischen Gebrauch zweier Schlagwörter mit Hervorhebung jeweils unterschiedlicher Merkmale/Eigenschaften des bezeichneten Sachverhalts in der Funktion der Einstellungskundgabe bezieht (Klein 1989, S. 20).

6 Die soziologische Vorurteilsforschung hat hier einen bedeutenden Beitrag geleistet. Standard ist stets Allport (1971).

7 „Ein Stereotyp ist [...] eine mentale Repräsentation im LZG [Langzeitgedächtnis], die als charakteristisch erachtete Merkmale / Eigenschaften eines Menschen bzw. einer Gruppe von Menschen abbildet und dabei durch grobe Generalisierung bzw. Simplifizierung eine reduzierte, verzerrte oder falsche Konzeptualisierung des Repräsentierten darstellt.“ (Schwarz-Friesel 2007, S. 337f.) Stereotype sind evident ,in diskriminierender, abwertender und ausgrenzender Funktion“ (Schwarz-Friesel 2007, S. 327) und drücken, indem sie versprachlichte Vorurteile sind, auch Emotionen aus, besser: emotional geprägte Haltungen.

8 Unter der Voraussetzung, dass Emotionen „Werte und Werterfahrungen konstituieren“, sind sie „intern repräsentierte und subjektiv erfahrbare Evaluationskategorien, die sich vom Individuum ich-bezogen introspektiv-geistig (als Gefühle) sowie körperlich registrieren lassen, deren Erfahrenswerte an eine positive oder negative Bewertung gekoppelt sind. Die Prozesse der Bewertung betreffen Einschät- 
zungen, mit denen ein Individuum entweder ein eigenes Körperbefinden, seine Handlungsimpulse, seine kognitiven Denkinhalte oder allgemein Umweltsituationen [...] beurteilt." (Schwarz-Friesel 2007, S. 73) Hinsichtlich der Konzeption von Menschenbildern haben Emotionen eine zentrale Funktion. Sie „steuern maßgeblich unsere Denkund Handlungsprozesse, determinieren die Interpretation und Evaluation von Mitmenschen und Situationen, erleichtern, erschweren oder hemmen unsere Lernprozesse und haben auf unsere Erinnerungsprozesse einen erheblichen Einfluss." (Schwarz-Friesel 2007, S. 4)

9 „Wie man jeweils - zu bestimmten Zeiten, in bestimmten Gruppen - über Menschen dachte, welche Selbst- und Fremdverständnisse man hatte, also welche Menschenbilder man gehabt hat - diese Fragen stellt man sich in der Mentalitätsgeschichte [...] besonders häufig, allerdings bezogen immer auf Spezialprobleme wie die Frage nach den Nationalstereotypen bei bestimmten Gruppen zu bestimmten Zeiten.“ (Hermanns 1994, S. 62f.)

10 „In den Auf- und Abwertungsstrategien wird die jeweilige Position / Person entweder direkt bewertet, oder sie wird argumentativ oder assoziativ mit Sachverhalten von eindeutiger Wertigkeit verknüpft. Dabei besteht die Tendenz zu polarisierender Werteverteilung auf die eigene und die konkurrierende Position - meist ohne Zwischenwerte." (Klein 1998, S. 378)

${ }^{11}$ Nach Hermanns (1994, S. 43).

${ }^{12}$ Erkennbar ist diese Tatsache der Grund für „eine bis heute anhaltende Diskussion über die Frage, ob es sich bei der anhaltenden Relevanz von Ethnizität um ein historisches Relikt handelt, das bei der vollständigen Durchsetzung gesellschaftlicher Modernisierung automatisch verschwindet, oder ob ethnische Differenzierung stattdessen ein konstitutives Strukturprinzip moderner Gesellschaften darstellt." (Müller 2007, S. 512)

${ }^{13}$ In der soziologischen Forschung ist die "Teilungsdimension ,Ethnie' [...] ein zentrales Konzept [...]. In der Regel werden ethnische Differenzen dabei als sozial konstruierte Wissensbestände zur Klassifikation von Personen verstanden, die regelmäßig Auswirkung auf die soziale Ordnung zwischen Menschen und Menschengruppen implizieren. [...] bei der ethnischen Zugehörigkeit [handelt es sich] um ein Produkt sozialer Interaktionen [...] Dieses ,doing ethnicity lässt sich in Attribution und Darstellung ethnischer Zugehörigkeiten unterteilen, die präreflexiv von den Interaktionsteilnehmern wechselseitig vorgenommen werden, je nach Situationskontext variieren können und in der ständigen Reproduktion ethnischer Differenzen resultieren." (Müller 2007, S. 512)
${ }^{14}$ Z. B.: Dabei müssen die nationalen Interessen und das Wohl des deutschen Volkes im Mittelpunkt stehen. (AfD 2016, S. 20)

${ }^{15}$ Mit dieser Haltung propagiert die AfD die Ethnonation, das heißt, ethnische Herkunft ist nationales Zugehörigkeits- bzw. Ausschlusskriterium. Der Gegenbegriff ist Staatsbürgernation, womit ein Gemeinwesen bezeichnet wird, das aus den Staatsangehörigen, unabhängig von ihrer Herkunft gebildet wird: „Innerhalb der vergangenen 200 Jahre lässt sich [...] auch innerhalb klassischer Staatsbürgernationen, wie z. B. Frankreich und den USA, eine zunehmende Ethnisierung der Mitgliedschaftskriterien für die Staatsbürgerschaft feststellen. [...] Im Fall der ,Ethnonation' fungiert die ethnische Herkunft also als Legitimation für die Staatsgründung." (Müller 2007, S. 514)

${ }_{16}$ Deontische Wörter (Hermanns 1986, S. 190ff.) sind ein Sollen bezeichnende Wörter (explizit: Pflicht, Sünde, können, böse etc.; implizit: Unkraut - 'vernichten').

${ }^{17}$ Die negativ-deontische Bedeutung eines Wortes wie Unkraut lautet etwa 'eine Pflanze, die unerwünscht, daher zu entfernen ist'. Die positiv-deontische Bedeutung eines Wortes wie Freiheit lautet etwa 'ein Zustand, der sehr erwünscht, daher unbedingt zu erreichen ist'.

18 „Das Phänomen und die Möglichkeit der multikulturellen Gesellschaft werden vornehmlich im Kontext der westlichen Gesellschaften diskutiert, und der Diskurs um die Menschenrechte erweckt auf den ersten Blick den Anschein eines Kulturkampfes, in dem der Westen seine Werte gegen die ,Anderen' zu verteidigen sucht. [...] Dabei werden allzu häufig und [...] leichtfertig dichotomische Gegensätze zwischen z. B. dem ,Westen' und dem ,Rest' aufgebaut, die den heutigen globalen Austauschbeziehungen nicht gerecht werden können. Während die politischen, ökonomischen und kulturellen Situationen der Gegenwart eine differenzierte und prozesshafte Vorgehensweise erfordern, beharrt der Diskurs um Menschenrechte weitgehend auf der herkömmlichen Denkweise der Identität, nach der eine kulturelle Einheit klar abgegrenzt sowie statisch in sich ruhend und homogen verstanden wird." (Shimada 2004, S. 474) 
${ }^{19}$ Weniger evident, wiewohl ebenfalls belegbar, ist der bedeutungsverwandte Gebrauch von

Nation / national: Forderung nach nationalen SoftwareEntwicklungen (AfD 2016, S. 57);

Wir stehen für die Freiheit der europäischen Nationen von fremder Bevormundung. Rechtsstaatliche Strukturen, wirtschaftlicher Wohlstand und ein stabiles, leistungsgerechtes Sozialsystem gehören in die nationale Verantwortung. (AfD 2016, S. 10);

Die AfD tritt deshalb dafür ein, zur deutschen Außenund Sicherheitspolitik eine langfristige ressortübergreifende Gesamtstrategie zu erarbeiten und umzusetzen. Dabei müssen die nationalen Interessen und das Wohl des deutschen Volkes im Mittelpunkt stehen (AfD 2016, S. 20).

${ }^{20}$ „Von kulturwissenschaftlichen Ansätzen entwickelte [...] Begriffe des Nationalismus als kontinuierliche Praxis der politischen Gemeinschaftsimagination, als immer wieder neue Konstruktion einer gedachten Ordnung und als Erfindung kollektiver Traditionen“ (Geulen 2004, S. 451).

${ }^{21}$ Diesen Terminus bewertet die AfD als unzutreffend und bietet eine Differenzierung an, die die Kriminalisierung derjenigen zur Voraussetzung hat, die sich nicht vor Krieg und Verfolgung retten müssen und die als „irreguläre Migranten" kategorisiert werden:

Die unzutreffende Bezeichnung „Flüchtling“ für fast alle Menschen, die irregulär nach Deutschland einreisen, um hier zu bleiben, ist Ausdruck der Verfehltheit dieser Politik. Es ist demgegenüber notwendig, zwischen politisch Verfolgten und (Kriegs-)Flüchtlingen, die unmittelbar vor ihrer Einreise echten, kriegsbedingten Gefahren ausgesetzt waren, einerseits und irregulären Migranten andererseits zu unterscheiden. Echte Flüchtlinge will auch die AfD schützen, solange die Fluchtursache im Heimatland andauert. Irreguläre Migranten aber, die, anders als Flüchtlinge, nicht verfolgt werden, können keinen Flüchtlingsschutz beanspruchen. (AfD 2016, S. 47)

${ }^{22}$ Es wurden allerdings angesichts der zeitweiligen Spaltung der Fraktion im Landesparlament von Baden-Württemberg aufgrund antisemitischer Äußerungen eines Fraktionsmitglieds Forderungen nach partieller Überwachung durch den Verfassungsschutz gestellt.

\section{Quellen}

AfD (2016): Grundsatzprogramm der Alternative für Deutschland. Vorlage zum Bundesparteitag am 30.04.2016/01.05. 2016. Zit. nach <https://www.alternativefuer.de/wp-content/uploads/sites/111/2016/03/Leitantrag-Grundsatzprogramm-AfD.pdf>; letzter Zugriff: 19.12.2016.

DVP (1920): Grundsätze der Deutschnationalen Volkspartei. In: Treue, Wolfgang (Hg.) (1968): Deutsche Parteiprogramme seit 1861 (= Quellensammlung zur Kulturgeschichte 3). 4. Aufl. Göttingen: Musterschmidt, S. 120-127.

Hitler, Adolf (1923): Rede auf einer Kundgebung in München am 21. August 1923. In: Michaelis, Herbert et al. (Hg.): Die Weimarer Republik / Das kritische Jahr 1923 (= Ursachen und Folgen. Vom deutschen Zusammenbruch 1918 und 1945 bis zur staatlichen Neuordnung Deutschlands in der Gegenwart. Eine Urkunden- und Dokumentensammlung zur Zeitgeschichte 5), Berlin: Dokumenten-Verlag Dr. Herbert Wendler \& Co, S. 181-184.

NSDAP (1920): Programm der Nationalsozialistischen Deutschen Arbeiterpartei. In: Treue, Wolfgang (Hg.) (1968): Deutsche Parteiprogramme seit 1861 (= Quellensammlung zur Kulturgeschichte 3). 4. Aufl. Göttingen: Musterschmidt. S. 156-159.

\section{Literatur}

Allport, Gordon W. (1971): Die Natur des Vorurteils. Hrsg. und kommentiert von Carl Friedrich Graumann. Köln: Kiepenheuer \& Witsch.

Burkhardt, Armin (2008): Deutsche Sprachgeschichte und politische Geschichte. In: Handbücher zur Sprach- und Kommunikationswissenschaft 2/1. Sprachgeschichte. 2. Aufl. Berlin / New York: de Gruyter, S. 98-122.

Busse, Dietrich (1997): Das Eigene und das Fremde. Zu Funktion und Wirkung einer diskurssemantischen Grundfigur. In: Jung, Matthias / Wengeler, Martin / Böke Karin (Hg.): Die Sprache des Migrationsdiskurses. Das Reden über "Ausländer" in Medien, Politik und Alltag. Opladen: Westdeutscher Verlag, S. 17-35.

Dieckmann, Walther (1975): Sprache in der Politik. Einführung in die Pragmatik und Semantik der politischen Sprache. 2. Aufl. Heidelberg: Winter.

Geulen, Christian (2004): Nationalismus als kulturwissenschaftliches Forschungsfeld. In: Jaeger, Friedrich / Rüsen, Jörn (Hg.): Handbuch der Kulturwissenschaften 3. Themen und Tendenzen. Stuttgart: Metzler, S. 439-457. 
Hermanns, Fritz (1986): Appellfunktion und Wörterbuch. Ein lexikographischer Versuch. In: Kämper, Heidrun/Linke, Angelika / Wengeler, Martin (Hg.)(2012): Fritz Hermanns. Der Sitz der Sprache im Leben. Beiträge zu einer kulturanalytischen Linguistik. Berlin / Boston: de Gruyter, S. 181-206.

Hermanns, Fritz (1989): Deontische Tautologien. Ein linguistischer Beitrag zur Interpretation des Godesberger Programms (1959) der Sozialdemokratischen Partei Deutschlands. In: Klein, Josef (Hg.): Politische Semantik. Bedeutungsanalytische und sprachkritische Beiträge zur politischen Sprachverwendung. Opladen: Westdeutscher Verlag, S. 69-149.

Hermanns, Fritz (1994): Linguistische Anthropologie. In: Kämper, Heidrun / Linke, Angelika / Wengeler, Martin (Hg.) (2012): Fritz Hermanns. Der Sitz der Sprache im Leben. Beiträge zu einer kulturanalytischen Linguistik. Berlin / Boston: de Gruyter, S. 37-66.

Hermanns, Fritz (1995): Deutsch und Deutschland. In: Kämper, Heidrun/Linke, Angelika/Wengeler, Martin (Hg.) (2012): Fritz Hermanns. Der Sitz der Sprache im Leben. Beiträge zu einer kulturanalytischen Linguistik. Berlin/Boston: de Gruyter, S. 295-310.

Klein, Josef (1989): Wortschatz, Wortkampf, Wortfelder in der Politik. In: Klein, Josef (Hg.): Politische Semantik. Bedeutungsanalytische und sprachkritische Beiträge zur politischen Sprachverwendung. Opladen: Westdeutscher Verlag, S. 3-50.

Klein, Josef (1998): Politische Kommunikation als Sprachstrategie. In: Jarren, Otfried / Sarcinelli, Ulrich / Saxer, Ulrich (Hg.): Politische Kommunikation in der demokratischen Gesellschaft. Ein Handbuch. Wiesbaden: Westdeutscher Verlag, S. 376-395.

Klein, Josef (2008): Textsorten im Bereich politischer Institutionen. In: Handbücher zur Sprach- und Kommunikationswissenschaft 16/1. Text- und Gesprächslinguistik. Berlin / New York: de Gruyter. S. 732-755.

Liedtke, Frank (1996): Stereotypensemantik, Metapherntheorie und Illokutionsstruktur. Die Analyse politischer Leitvokabeln in linguistischer Perspektive. In: Böke, Karin / Liedtke, Frank / Wengeler Martin (Hg.): Politische Leitvokabeln in der Adenauer-Ära. Berlin / New York: de Gruyter, S. 1-17.

Loewenstein, Bedrich (2001): „Am deutschen Wesen ...”. In: François Etienne / Schulze, Hagen (Hg.): Deutsche Erinnerungsorte. 1. Bd. München: Beck, S. 290-304.

Lübbe, Hermann (1975): Der Streit um Worte. Sprache und Politik. In: Kaltenbrunner, Gerd-Klaus (Hg.): Sprache und Herrschaft. Die umfunktionierten Wörter. Freiburg u. a.: Herder, S. 87-111.
Müller, Marion (2007): Ethnizität. In: Schützeichel, Rainer (Hg.): Handbuch Wissenssoziologie und Wissensforschung. Konstanz: UVK-Verl.-Ges., S. 512-520.

Schwarz-Friesel, Monika (2007): Sprache und Emotion. 2. Aufl. Tübingen / Basel: A. Francke.

Shimada, Shingo (2004): Politik zwischen Differenz und Anerkennung: Multikulturalismus und das Problem der Menschenrechte. In: In: Jaeger, Friedrich / Rüsen, Jörn (Hg.). Handbuch der Kulturwissenschaften 3. Themen und Tendenzen. Stuttgart: Metzler, S. 474-488.

\section{Bildnachweise}

S. 2: $\quad$ https://commons.wikimedia.org/wiki/ File:Alternative_f $\%$ C3\%BCr_Deutschland_-hand_bills,_flyer.jpg

S. 4: $\quad$ https://commons.wikimedia.org/wiki/ Category:Grundgesetz\#/media/File:Stamp_ Germany_1999_MiNr2050_Grundgesetz.jpg

S. 8: $\quad$ shutterstock_ 527115829

S. 9: pixelio_459162

S. 10-11: shutterstock_228969187

S. 14: shutterstock_ 569554777

S. 15: $\quad$ shutterstock_ 555114967

S. 17: $\quad$ shutterstock_ 481841314

Dies ist eine bearbeitete Fassung des Aufsatzes, der zuerst in Heft 1/2017 von Aptum. Zeitschrift für Sprachkritik und Sprachkultur erschienen ist. 